\title{
MONGE-AMPĖRE MEASURES OF PLURISUBHARMONIC EXHAUSTIONS ASSOCIATED TO THE LIE NORM OF HOLOMORPHIC MAPS
}

\author{
RAGNAR SIGURDSSON AND AUĐUNN SKÚTA SNÆBJARNARSON \\ Dedicated to the memory of Professor Józef Siciak
}

\begin{abstract}
In this paper we derive formulas for the Monge-Ampère measures of functions of the form $\log |\Phi|_{c}$, where $\Phi$ is a holomorphic map on a complex manifold $X$ of dimension $n$ with values in $\mathbb{C}^{n+1} \backslash\{0\}$ and $|\cdot|_{c}$ is the Lie norm on $\mathbb{C}^{n+1}$.
\end{abstract}

MSC (2010): Primary 32U05; Secondary 32Q28, 32U10, 32W20

\section{INTRODUCTION}

This paper grew out of a study of a generalization of the Bernstein-WalshSiciak theorem to Stein manifolds by the second author [22], where the problem of constructing examples of plurisubharmonic (psh) exhaustion functions which are maximal outside a compact set appears naturally. The main result of the paper is the following.

Theorem 1.1. Let $\Phi=\left(\Phi_{0}, \ldots, \Phi_{n}\right): X \rightarrow \mathbb{C}^{n+1} \backslash\{0\}$ be a holomorphic map on a complex manifold of dimension $n$. The function $\log |\Phi|_{c}$, where $|\cdot|_{c}$ denotes the Lie norm on $\mathbb{C}^{n+1}$, is psh on $X$ and a maximal $C^{\infty}$ function on $\Phi^{-1}\left(\mathbb{C}^{n+1} \backslash \mathbb{C R}^{n+1}\right)$, where $\mathbb{C R}^{n+1}=\left\{\alpha a ; \alpha \in \mathbb{C}, a \in \mathbb{R}^{n+1}\right\}$. The MongeAmpère measure $\left(d d^{c}\left(\log |\Phi|_{c}\right)\right)^{n}$ has no mass on the set

$$
\mathcal{A}_{\Phi}=\{z \in X ; \operatorname{rank} d \Phi(z)<n \text { or } \Phi(z) \in \operatorname{range} d \Phi(z)\} .
$$

If $M=\Phi^{-1}\left(\mathbb{C R}^{n+1}\right) \backslash \mathcal{A}_{\Phi}$ is non-empty, then it is an n-dimensional real analytic manifold and $\left(d d^{c}\left(\log |\Phi|_{c}\right)\right)^{n}$ is the current of integration along $M$ of the n-form

$$
C_{n}\left(\Phi_{0}^{2}+\cdots+\Phi_{n}^{2}\right)^{-\frac{(n+1)}{2}} \sum_{j=0}^{n}(-1)^{j+1} \Phi_{j} d \Phi_{0} \wedge \cdots \wedge \widehat{d \Phi_{j}} \wedge \cdots \wedge d \Phi_{n}
$$

Date: March 20, 2019. 
where $C_{n}=(-1)^{\frac{n(n-1)}{2}} n ! \Omega_{n}, \Omega_{n}$ is the volume of the unit-ball in $\mathbb{R}^{n}$, and the holomorphic square root of the function $\Phi_{0}^{2}+\cdots+\Phi_{n}^{2}$ is chosen with the same argument as the vector $\Phi$ in $\mathbb{C R}^{n+1}$.

The Monge-Ampère measure of a $C^{2}$ psh function $v$ on an open subset of $\mathbb{C}^{n}$ is defined as

$$
\left(d d^{c} v\right)^{n}=\underbrace{\left(d d^{c} v\right) \wedge \cdots \wedge\left(d d^{c} v\right)}_{n \text { times }}=4^{n} n ! \operatorname{Det}\left(\frac{\partial^{2} v}{\partial z_{j} \partial \bar{z}_{k}}\right)_{j, k} d V,
$$

where $d^{c}=i(\bar{\partial}-\partial)$ and $d V=\bigwedge_{j=1}^{n} \frac{i}{2} d z_{j} \wedge d \bar{z}_{j}$ is the standard Euclidean volume form on $\mathbb{C}^{n}$. By the fundamental work of Bedford and Taylor [5, 6] one can define the Monge-Ampère measure of a $L_{\text {loc }}^{\infty}$ psh function $v$ as

$$
\left(d d^{c} v\right)^{n}:=\lim _{k \rightarrow \infty}\left(d d^{c} v_{k}\right)^{n}
$$

where the limit is taken in the weak* topology of measures and $\left(v_{k}\right)$ is any sequence of smooth psh functions decreasing to $v$.

The Lie norm on $\mathbb{C}^{N}$ is the largest norm on $\mathbb{C}^{N}$ which extends the Euclidean norm $|\cdot|$ on $\mathbb{R}^{N}$ to a complex norm on $\mathbb{C}^{N}$. It thus dominates the standard Hermitian norm on $\mathbb{C}^{N}$. It is given by an explicit formula in Proposition 2.1, which shows that it is a $C^{\infty}$ function on $\mathbb{C}^{N} \backslash \mathbb{C R}^{N}$. By Bos, Levenberg, Ma'u, and Piazzon [9] the 0 eigenspace of the Levi matrix of $\log |\cdot|_{c}$ on $\mathbb{C}^{N}$ at every $\zeta \neq 0$ is spanned by $\zeta$ and $\bar{\zeta}$. This is the case $\varepsilon=0$ in Theorem 6.1 below. These vectors are linearly independent if and only if $\zeta$ is in $\mathbb{C}^{N} \backslash \mathbb{C} \mathbb{R}^{N}$, so the kernel of the Levi matrix of the function $\log |\cdot|_{c}$ is two dimensional at every $\zeta \in \mathbb{C}^{N} \backslash \mathbb{C R}^{N}$. Hence $\log |\Phi|_{c}$ is maximal on $\Phi^{-1}\left(\mathbb{C}^{n+1} \backslash \mathbb{C R}^{n+1}\right)$ for every holomorphic map $\Phi: X \rightarrow \mathbb{C}^{n+1}$ on a manifold of dimension $n$. This is the advantage of the Lie norm rather than, say, the usual Euclidean norm for constructing maximal psh functions. For a general $\Phi: X \rightarrow \mathbb{C}^{n+1}$ the function $\log |\Phi|$ is not maximal on any open subset of $X$.

Recall that a Stein manifold $X$ is said to be parabolic if every bounded psh function on $X$ is constant, it is said to be $S$-parabolic if there exists a psh exhaustion on $X$ which is maximal outside a compact subset of $X$. Such an exhaustion is called a parabolic potential or a special psh exhaustion function. The Stein manifold is said to be $S^{*}$-parabolic if there exists a continuous parabolic potential on $X$. See Aytuna and Sadullaev [1, 2, 3] for a study of these classes of manifolds. 
In order to understand when we can expect to find a parabolic potential of the form $\log |\Phi|_{c}$, let us look at a slightly more general problem. Let $\Phi: X \rightarrow Y$ be a holomorphic map on a manifold $X$ of dimension $n$ into a manifold $Y$ of dimension $N$ and let $h$ be a psh function on $Y$. If $h$ is an exhaustion and $\Phi$ is a proper map, then $h \circ \Phi$ is an exhaustion on $X$. If $h$ is a $C^{2}$ function on an open subset $V$ of $Y$ then $h$ is maximal on $V$ if and only if the Levi matrix of $h$ has eigenvalue 0 at every point $\zeta \in V$. Recall that the Levi form of $h$ is the Hermitian form $w \mapsto \mathcal{L}_{h}(\zeta, w)=\sum_{j, k=1}^{N} h_{j \bar{k}}(\zeta) w_{j} \bar{w}_{k}$, where the Levi matrix $\left(h_{j \bar{k}}(\zeta)\right)_{j, k=1}^{N}$, is given in local coordinates $\zeta=\left(\zeta_{1}, \ldots, \zeta_{N}\right)$ as $h_{j \bar{k}}=\partial^{2} h / \partial \zeta_{j} \partial \bar{\zeta}_{k}$ and $\left(w_{1}, \ldots, w_{N}\right)$ are the coordinates of the tangent vector $w$ at $\zeta$ with respect to the basis $\left(\partial / \partial \zeta_{1}, \ldots, \partial / \partial \zeta_{N}\right)$. We have

$$
\mathcal{L}_{h \circ \Phi}(z ; w)=\mathcal{L}_{h}(\Phi(z), d \Phi(z)(w)), \quad z \in \Phi^{-1}(V), w \in T_{z} X
$$

From this equation we see that if for every $z \in \Phi^{-1}(V)$ there exists a tangent vector $w \neq 0$ of $X$ at $z$ which is mapped by $d \Phi(z)$ to the kernel of the Levi matrix of $h$ at $\Phi(z)$, then $h \circ \Phi$ is maximal on $\Phi^{-1}(V)$. If at every $\zeta \in V$ the kernel of the Levi matrix of $h$ at $\zeta$ has dimension at least $k \geq 1$ and $N=n+k-1$, then it follows by the rank-nullity theorem from linear algebra that $h \circ \Phi$ is maximal on $\Phi^{-1}(V)$.

Our observations can now be summarized as follows:

Corollary 1.2. If $X$ is a Stein manifold of dimension $n$, and there exists a proper holomorphic map $\Phi: X \rightarrow \mathbb{C}^{n+1}$, such that $\Phi^{-1}\left(\mathbb{C R}^{n+1}\right)$ is compact, then $X$ is $S^{*}$-parabolic. More generally, if there exists a proper holomorphic map $\Phi: X \rightarrow \mathbb{C}^{N}, N \geq n$, such that $\Phi^{-1}\left(\mathbb{C R}^{N}\right)$ is compact and such that for every $z \in X$ there exists a tangent vector $w \neq 0$ at $z$ which is mapped by the derivative $d \Phi(z)$ at $z$ into the span of $\Phi(z)$ and $\overline{\Phi(z)}$, then $X$ is $S^{*}$-parabolic.

There is an extensive literature on the existence of proper holomorphic maps on Stein manifolds, see e.g., Forstnerič [14].

The plan of the paper is as follows. In Section 2 we review the general theory of the cross norm $\|\cdot\|_{c}$ on the complexification $V_{\mathbb{C}}$ of a real normed space $(V,\|\cdot\|)$. For an inner product space $(V,\langle\cdot, \cdot\rangle)$ with norm $|\cdot|$ we derive a simple formula for the cross norm, $|\zeta|_{c}=|a|+|b|$, where the vector $\zeta \in V_{\mathbb{C}}$ is represented as $\zeta=e^{i \theta}(a+i b)$, with $\theta \in \mathbb{R}, a, b \in V,\langle a, b\rangle=0$, and $|b| \leq|a|$. From this formula we give a new proof of the explicit formulas for 
$|\zeta|_{c}$ originally proved by Drużkowski in [13]. If $|\cdot|$ is the Euclidean norm on $\mathbb{R}^{N}$, then $|\cdot|_{c}$ is called the Lie norm on $\mathbb{C}^{N}$.

In Section 3 we first take a look at the set $\mathbb{C R}^{N}$ from a few different viewpoints. Then we look at the representation of $\zeta \in \mathbb{C}^{N}$ as $\zeta=e^{i \theta}(a+i b)$ with $\theta \in \mathbb{R}, a, b \in \mathbb{R}^{N},\langle a, b\rangle=0$, and $|b| \leq|a|$, which is very useful in our calculations. We derive a formula for the pullback of the volume form $d V$ on $\mathbb{C}^{N}$ to the $2 N$-dimensional real manifold $\{(\theta, a, b) ;\langle a, b\rangle=0,|b|<|a|\}$. This formula is applied in Section 7 for calculating weak limits of MongeAmpère measures.

In Sections 4 and 5 we apply Theorem 1.1 to calculate the SiciakZakharyuta extremal function and the corresponding equilibrium measure for a few compact subsets $K$ of $\mathbb{R}^{n} \subset \mathbb{C}^{n}$. The extremal function has been extensively studied for several decades and, in particular, authors have shown interest in the case when $K$ is a compact subset of $\mathbb{R}^{N}$ [4, 17, ㅇ,, 10 , 11, 12, 18]. Among our examples is a result that motivated this study [9].

As a preparation for the proof of Theorem 1.1 we analyze in Section 6. the Levi form of the maximal function $h_{\varepsilon}=\log v_{\varepsilon}$, where the family of functions, $v_{\varepsilon}(\zeta)=(|a|+\varepsilon|b|)^{\frac{1}{2}}+(|b|+\varepsilon|a|)^{\frac{1}{2}}, \varepsilon \geq 0$, regularizes the Lie norm $v(\zeta)=|\zeta|_{c}=|a|+|b|$. Finally, in Section 7 we complete the proof of Theorem 1.1 by calculating the Monge-Ampère measure $\left(d d^{c}\left(\log |\Phi|_{c}\right)\right)^{n}$. as the weak limit of $\left(d d^{c}\left(\log v_{\varepsilon} \circ \Phi\right)\right)^{n}$

Acknowledgment. This work was partially supported by by The Icelandic Centre for Research (Rannís), grant no. 152572-052, and a doctoral grant from The University of Iceland Research Fund. The authors would like to thank the anonymous referee for comments and suggestions that led to considerable improvements of the paper.

\section{LIE NORM}

The Lie norm on $\mathbb{C}^{N}$ is a special case of an extension of a norm on a real vector space $V$ to a complex norm on its complexification $V_{\mathbb{C}}=\mathbb{C} \otimes_{\mathbb{R}} V$, i.e., the extension satisfies $\|\alpha \zeta\|=|\alpha|\|\zeta\|$ for $\alpha \in \mathbb{C}$ and $\zeta \in V_{\mathbb{C}}$. There are a few different ways of introducing the complexification, see Munoz, Sarantopoulos, and Tonge [21]. One is to define $V_{\mathbb{C}}$ as $V \times V$ with usual 
addition and complex multiplication $(\alpha+i \beta)(\xi, \eta)=(\alpha \xi-\beta \eta, \beta \xi+\alpha \eta)$ where $\alpha, \beta \in \mathbb{R}$ and $\xi, \eta \in V$. We have $(0, \eta)=i(\eta, 0)$, so if we identify the real vector $\xi$ in $V$ with $(\xi, 0)$ in $V_{\mathbb{C}}$, then we can write every vector $\zeta=(\xi, \eta)$ as $\zeta=\xi+i \eta$. Thus $V$ is a real subspace of $V_{\mathbb{C}}$ and $V_{\mathbb{C}}=V \oplus i V$. The vectors $\xi$ and $\eta$ are called the real and imaginary parts of $\zeta$, denoted by $\operatorname{Re} \zeta$ and $\operatorname{Im} \zeta$.

In 21] it is shown that every norm $\|\cdot\|$ on $V$ can be extended to a complex norm on $V_{\mathbb{C}}$. If we have such an extension and $\zeta=\sum_{j=1}^{k} \alpha_{j} \xi_{j}$ is a vector in $V_{\mathbb{C}}$, where $\alpha_{j} \in \mathbb{C}$ and $\xi_{j} \in V$, then the triangle inequality implies $\|\zeta\| \leq \sum_{j=1}^{k}\left|\alpha_{j}\right|\left\|\xi_{j}\right\|$. For $\zeta \in V_{\mathbb{C}}$ we define

$$
\|\zeta\|_{c}=\inf \left\{\sum_{j=1}^{k}\left|\alpha_{j}\right|\left\|\xi_{j}\right\| ; \zeta=\sum_{j=1}^{k} \alpha_{j} \xi_{j}, \alpha_{j} \in \mathbb{C}, \xi_{j} \in V, k \in \mathbb{N}^{*}\right\} .
$$

Since we can extend every norm on $V$ to a complex norm on $V_{\mathbb{C}}$, it is easy to see that $\zeta \mapsto\|\zeta\|_{c}$ is a norm and moreover it is the largest complex norm on $V_{\mathbb{C}}$ which extends $\|\cdot\|$ from $V$. The norm $\|\cdot\|_{c}$ on $V_{\mathbb{C}}$ is called the crossnorm of $\|\cdot\|$. If $|\cdot|$ denotes the Euclidean norm on $\mathbb{R}^{N}$ then the cross norm $|\cdot|_{c}$ is called the Lie norm on $\mathbb{C}^{N}$.

The set $\mathbb{C} V=\left\{\alpha \xi \in V_{\mathbb{C}} ; \alpha \in \mathbb{C}, \xi \in V\right\}=\left\{e^{i \theta} \xi \in V_{\mathbb{C}} ; \theta \in \mathbb{R}, \xi \in V\right\}$ consisting of all vectors in $V_{\mathbb{C}}$ with parallel real and imaginary parts is important in our calculations. If $\|\cdot\|$ extends from $V$ to a complex norm on $V_{\mathbb{C}}$, also denoted by $\|\cdot\|$, then we denote the distance from $\zeta \in V_{\mathbb{C}}$ to $\mathbb{C} V$ by $d(\zeta, \mathbb{C} V)=\inf \left\{\left\|\zeta-e^{i \theta} \xi\right\| ; \theta \in \mathbb{R}, \xi \in V\right\}$. For $\zeta=e^{i \theta} \xi \in \mathbb{C} V$ we have $\|\zeta\|=\|\xi\|$.

Assume from now on that $V$ is an inner product space with norm $\xi \mapsto$ $|\xi|=\langle\xi, \xi\rangle^{\frac{1}{2}}$, where $(x, \xi) \mapsto\langle x, \xi\rangle$ is an inner product on $V$. The bilinear form on $V$ has a unique extension to a symmetric $\mathbb{C}$-bilinear form on $V_{\mathbb{C}}$ by the formula

$$
\langle x+i y, \xi+i \eta\rangle=\langle x, \xi\rangle-\langle y, \eta\rangle+i\langle x, \eta\rangle+i\langle y, \xi\rangle,
$$

and the Hermitian form $(z, \zeta) \mapsto\langle z, \bar{\zeta}\rangle$ generates an extension of the norm by the formula

$$
|\zeta|=\langle\zeta, \bar{\zeta}\rangle^{\frac{1}{2}}=\left(|\xi|^{2}+|\eta|^{2}\right)^{\frac{1}{2}}, \quad \zeta=\xi+i \eta \in V_{\mathbb{C}}
$$

Take $\zeta \in V_{\mathbb{C}}$ and $\theta \in \mathbb{R}$ such that $e^{-2 i \theta}\langle\zeta, \zeta\rangle$ is a positive real number. If $a=\operatorname{Re}\left(e^{-i \theta} \zeta\right)$ and $b=\operatorname{Im}\left(e^{-i \theta} \zeta\right)$, then $e^{-2 i \theta}\langle\zeta, \zeta\rangle=|a|^{2}-|b|^{2}+2 i\langle a, b\rangle \geq 0$, so we conclude that $\langle a, b\rangle=0$ and $|b| \leq|a|$. 
Proposition 2.1. If $\zeta=\xi+i \eta=e^{i \theta}(a+i b), \theta \in \mathbb{R}, \xi, \eta, a, b \in V,\langle a, b\rangle=0$, and $|b| \leq|a|$, then

$$
|a|=\frac{1}{\sqrt{2}}\left(|\zeta|^{2}+|\langle\zeta, \zeta\rangle|\right)^{\frac{1}{2}} \quad \text { and } \quad|b|=\frac{1}{\sqrt{2}}\left(|\zeta|^{2}-|\langle\zeta, \zeta\rangle|\right)^{\frac{1}{2}} .
$$

The $|\cdot|$-distance from $\zeta$ to $\mathbb{C} V$ is $d(\zeta, \mathbb{C} V)=|b|$ and the cross norm is given by the formula

$$
\begin{aligned}
|\zeta|_{c} & =|a|+|b|=\left(|\zeta|^{2}-d(\zeta, \mathbb{C} V)^{2}\right)^{\frac{1}{2}}+d(\zeta, \mathbb{C} V) \\
& =\left(|\zeta|^{2}+\left(|\zeta|^{4}-|\langle\zeta, \zeta\rangle|^{2}\right)^{\frac{1}{2}}\right)^{\frac{1}{2}} \\
& =\left(|\zeta|^{2}+2\left(|\xi|^{2}|\eta|^{2}-\langle\xi, \eta\rangle^{2}\right)^{\frac{1}{2}}\right)^{\frac{1}{2}}
\end{aligned}
$$

If $V$ is a Hilbert space and $\langle\zeta, \zeta\rangle \neq 0$, then $e^{i \theta} a$ is a unique point in $\mathbb{C} V$ with minimal distance to $\zeta$. If $\langle\zeta, \zeta\rangle=0$, then $\left\{e^{i \theta} \operatorname{Re}\left(e^{-i \theta} \zeta\right) ; \theta \in \mathbb{R}\right\}=$ $\left\{\frac{1}{2}\left(\zeta+e^{2 i \theta} \bar{\zeta}\right) ; \theta \in \mathbb{R}\right\}$ is a circle consisting of all points in $\mathbb{C} V$ with minimal distance to $\zeta$.

Proof. Equations (2.4) follow from the fact that $|\zeta|^{2}=|a|^{2}+|b|^{2}$, and $|\langle\zeta, \zeta\rangle|=|a|^{2}-|b|^{2}$. For proving the first equality in 2.5 we observe that $\zeta=e^{i \theta} a+e^{i \theta} i b$ is one of the linear combinations in 2.1. , so we have $|\zeta|_{c} \leq|a|+|b|$ and equality holds if $b=0$. Assume that $b \neq 0$ and take $e_{a}=a /|a|$ and $e_{b}=b /|b|$. If $\zeta=\sum_{j=1}^{k} \alpha_{j} \xi_{j}$ is some other representation of $\zeta$ as in 2.1, then $a=\sum_{j=1}^{k} \operatorname{Re}\left(e^{-i \theta} \alpha_{j}\right) \xi_{j}, b=\sum_{j=1}^{k} \operatorname{Im}\left(e^{-i \theta} \alpha_{j}\right) \xi_{j}$, so the Cauchy-Schwarz inequality and Pythagoras' theorem give

$$
\begin{aligned}
|a|+|b|=\left\langle a, e_{a}\right\rangle & +\left\langle b, e_{b}\right\rangle=\sum_{j=1}^{k}\left\langle\operatorname{Re}\left(e^{-i \theta} \alpha_{j}\right) e_{a}+\operatorname{Im}\left(e^{-i \theta} \alpha_{j}\right) e_{b}, \xi_{j}\right\rangle \\
\leq & \sum_{j=1}^{k}\left|\operatorname{Re}\left(e^{-i \theta} \alpha_{j}\right) e_{a}+\operatorname{Im}\left(e^{-i \theta} \alpha_{j}\right) e_{b}\right|\left|\xi_{j}\right|=\sum_{j=1}^{k}\left|\alpha_{j}\right|\left|\xi_{j}\right| .
\end{aligned}
$$

This proves the first equality in 2.5 and the others follow from (2.4). The proof of the remaining statements is identical to the proof of Lemma 5.18 in $[16]$.

Observe that the last two expressions for the Lie norm in 2.5 were first proved by Drużkowski [13]. 


\section{Geometry of $\mathbb{C R}^{N}$}

From now on we let $\langle\cdot, \cdot\rangle$ denote the natural bilinear form on $\mathbb{C}^{N},\langle z, \zeta\rangle=$ $\sum_{j=1}^{N} z_{j} \zeta_{j}$, which means that the Hermitian form is $(z, \zeta) \mapsto\langle z, \bar{\zeta}\rangle$. We let $|\cdot|$ denote the Hermitian norm on $\mathbb{C}^{N}$ and $|\cdot|_{c}$ denote the Lie norm.

The set $\mathbb{C R}^{N}$ consists of all vectors in $\mathbb{C}^{N}$ with parallel real and imaginary parts. Since $\mathbb{C R}^{N} \backslash\{0\}$ is the inverse image of the real projective space $\mathbb{P}^{N-1}(\mathbb{R})$ under the natural map from $\mathbb{C}^{N} \backslash\{0\}$ it is a real analytic manifold of dimension $N+1$. Moreover, the parametrization

$$
\mathbb{R} \times\left(\mathbb{R}^{N} \backslash\{0\}\right) \ni(\theta, a) \mapsto e^{i \theta} a \in \mathbb{C R}^{N} \backslash\{0\}
$$

has an injective differential at every point and $\mathbb{C R}^{N} \backslash\{0\}$ is a fiber bundle over $\mathbb{S}^{1}$ with the projection

$$
\mathbb{C R}^{N} \backslash\{0\} \ni \zeta=e^{i \theta} a \mapsto \frac{\langle\zeta, \zeta\rangle}{|\zeta|^{2}}=e^{2 i \theta} \in \mathbb{S}^{1}
$$

and fiber $\mathbb{R}^{N} \backslash\{0\}$. We have $|\zeta|^{2}-|\langle\zeta, \zeta\rangle|=4\left(|\xi|^{2}|\eta|^{2}-\langle\xi, \eta\rangle^{2}\right)$ for every $\zeta=\xi+i \eta \in \mathbb{C}^{N}$, which shows that $\mathbb{C R}^{N}$ is a real algebraic variety,

$$
\mathbb{C R}^{N}=\left\{\xi+i \eta \in \mathbb{C}^{N} ;|\xi||\eta|=|\langle\xi, \eta\rangle|\right\}=\left\{\zeta \in \mathbb{C}^{N} ;|\zeta|^{2}=|\langle\zeta, \zeta\rangle|\right\} .
$$

If $\zeta \in \mathbb{C R}^{N}$ is written as $e^{i \theta} a$, then the argument $\theta$ is determined up to a multiple of $\pi$ and the vector $a$ up to a sign.

In Section 2 we have seen how useful the $(\theta, a, b)$ coordinates are for the calculation of the Lie norm. We are going to use these coordinates to parameterize the set $\left\{\zeta \in \mathbb{C}^{N} ;\langle\zeta, \zeta\rangle \neq 0\right\}$ and to express the standard volume form on $\mathbb{C}^{N}$,

$$
d V=\left(\frac{i}{2}\right)^{N} \bigwedge_{j=1}^{N} d z_{j} \wedge d \bar{z}_{j}
$$

with respect to these coordinates.

Proposition 3.1. The pullback of the volume form $d V$ on $\mathbb{C}^{N}$, under the map $(\theta, a, b) \mapsto e^{i \theta}(a+i b)$ to the $2 N$ dimensional real manifold

$$
L=\left\{(\theta, a, b) ; \theta \in \mathbb{R}, a, b \in \mathbb{R}^{N},\langle a, b\rangle=0,|b|<|a|\right\} \subset \mathbb{R}^{2 N+1}
$$

is given by the formula

$$
d V=-d \theta \wedge \sum_{j=1}^{N}\left(a_{j} \Lambda_{b_{j}}+b_{j} \Lambda_{a_{j}}\right)
$$


where the $2 N-1$ forms $\Lambda_{a_{j}}$ and $\Lambda_{b_{j}}$ are given by

$$
\begin{aligned}
& \Lambda_{a_{j}}=d a_{1} \wedge d b_{1} \wedge \cdots \wedge \widehat{d a}_{j} \wedge d b_{j} \wedge \cdots \wedge d a_{N} \wedge d b_{N} \\
& \Lambda_{b_{j}}=d a_{1} \wedge d b_{1} \wedge \cdots \wedge d a_{j} \wedge \widehat{d b}_{j} \wedge \cdots \wedge d a_{N} \wedge d b_{N}
\end{aligned}
$$

and $\widehat{d a}_{j}$ and $\widehat{d b}_{j}$ is the standard notation for omitted factors. On the open subset $L_{k}:=\left\{(\theta, a, b) \in L ; a_{k} \neq 0\right\}$ of $L$ we can express the volume form as

$$
d V=\frac{|b|^{2}}{a_{k}} d \theta \wedge \Lambda_{b_{k}}-d \theta \wedge \sum_{j=1}^{N} a_{j} \Lambda_{b_{j}}
$$

Proof. We have $z_{j}=e^{i \theta}\left(a_{j}+i b_{j}\right)$ and $\bar{z}_{j}=e^{-i \theta}\left(a_{j}-i b_{j}\right)$, so

$$
\begin{aligned}
& d z_{j}=i e^{i \theta}\left(\left(a_{j}+i b_{j}\right) d \theta-i d a_{j}+d b_{j}\right) \quad \text { and } \\
& d \bar{z}_{j}=-i e^{-i \theta}\left(\left(a_{j}-i b_{j}\right) d \theta+i d a_{j}+d b_{j}\right)
\end{aligned}
$$

and we get

$$
d z_{j} \wedge d \bar{z}_{j}=-2 i\left(-a_{j} d \theta \wedge d a_{j}-b_{j} d \theta \wedge d b_{j}+d a_{j} \wedge d b_{j}\right)
$$

Now equation 3.6 follows by wedging equation 3.10 over all $j \in\{1, \ldots, N\}$, and noticing that $\bigwedge_{j=1}^{N} d a_{j} \wedge d b_{j}=0$, which follows from the equation $a_{1} b_{1}+\cdots a_{N} b_{N}=0$. Furthermore, this equation implies that $b_{j} \Lambda_{a_{j}}=$ $-\frac{b_{j}^{2}}{a_{k}} \Lambda_{b_{k}}$ on $L_{k}$ for every $j$ and $k$. If we combine this with 3.6 , then 3.9 follows.

In some cases the variables $\theta, a_{1}, \ldots, a_{N}, b_{1}, \ldots, b_{N}$ on $L$ are inconvenient to work with because they are dependent through the equality $\langle a, b\rangle=0$. Therefore we define the set

$$
\tilde{L}=\left\{(\theta, a, \beta) \in \mathbb{R} \times \mathbb{R}^{N} \times \mathbb{R}^{N-1} ;|\beta|<|a|\right\}
$$

and present the following proposition.

Proposition 3.2. For each $m \in\{1, \ldots, N\}$ there exists a change of variables $L_{m}:=\left\{(\theta, a, b) \in L ; a_{m} \neq 0\right\} \rightarrow \tilde{L},(\theta, a, b) \mapsto(\theta, a, \beta)$ such that $|\beta|=|b|$ and

$$
\begin{gathered}
d V=-(-1)^{\frac{N(N+1)}{2}}\left(|a|-\frac{|\beta|^{2}}{|a|}\right) d \theta \wedge d V_{a} \wedge d V_{\beta}, \quad \text { where } \\
d V_{a}=d a_{1} \wedge \cdots \wedge d a_{N} \quad \text { and } \quad d V_{\beta}=d \beta_{1} \wedge \cdots \wedge d \beta_{N-1} .
\end{gathered}
$$

Proof. For each $a$ with $a_{m} \neq 0$ we can define an orthogonal matrix $U_{a}=$ $\left(u_{j, k}(a)\right)$ whose last column is parallel to $a$ and the entries of the matrix $U_{a}$ are smooth as functions of $a$. This can be done by applying the GramSchmidt method on the basis $\left\{a, e_{1}, \ldots, e_{N}\right\} \backslash\left\{e_{m}\right\}$, where $\left\{e_{1}, \ldots, e_{N}\right\}$ 
is the standard basis. Now we define the change of variables $L_{m} \rightarrow \tilde{L}$, $(\theta, a, b) \mapsto(\theta, a, \beta)$ by

$$
\left(\beta_{1}, \ldots, \beta_{N-1}, 0\right)^{t}=U_{a}^{-1}\left(b_{1}, \ldots, b_{N}\right)^{t} .
$$

Since $U_{a}$ is orthogonal its cofactors are given by the equation $C_{j, k}=u_{j, k}$, in particular we have $C_{j, N}=a_{j} /|a|$. Therefore, for any $s \in\{1, \ldots, N\}$, we have

$$
\begin{aligned}
(-1)^{\frac{N(N-1)}{2}} \Lambda_{b_{s}}=(-1)^{s+N} d a_{1} \wedge \cdots \wedge d a_{N} \wedge d b_{1} \wedge \cdots \wedge \widehat{d b}_{s} \wedge \cdots \wedge d b_{N} \\
=(-1)^{s+N} d V_{a} \bigwedge_{j=1, j \neq s}^{N}\left(\sum_{k=1}^{N-1}\left(u_{j, k} d \beta_{k}+\beta_{k} d u_{j, k}\right)\right) \\
=(-1)^{s+N} d V_{a} \bigwedge_{j=1, j \neq s}^{N}\left(\sum_{k=1}^{N-1} u_{j, k} d \beta_{k}\right)=C_{s, N} d V_{a} \wedge d V_{\beta} \\
=\frac{a_{s}}{|a|} d V_{a} \wedge d V_{\beta}
\end{aligned}
$$

The result follows by combining equation 3.11 with equation 3.9 .

\section{Applications}

In this section we apply Theorem 1.1 to give explicit formulas for the SiciakZakharyuta extremal functions and the corresponding equilibrium measures for a few examples of compact sets. We have grouped the examples into three classes; namely we consider compact subsets of the Euclidean space $\mathbb{C}^{n}$, the projective space $\mathbb{P}^{n}(\mathbb{C})$, and the complex torus $\mathbb{C}^{n} / \mathbb{Z}^{n}$. For the rest of the section we write $\mathbb{R}_{+}:=[0, \infty)$.

Recall that the extremal function of a compact set $K \subset \mathbb{C}^{n}$ is defined by the equation

$$
V_{K}(z)=\sup \left\{u(z) ; u \in L\left(\mathbb{C}^{n}\right),\left.u\right|_{K} \leq 0\right\}, \quad z \in \mathbb{C}^{n},
$$

where $L\left(\mathbb{C}^{n}\right)$ is the Lelong class of all psh functions $u$ on $\mathbb{C}^{n}$ satisfying the growth condition $u(z)-\log |z| \leq O(1)$ as $|z| \rightarrow \infty$. The Monge-Ampère measure $\left(d d^{c} V_{K}\right)^{n}$ is called the equilibrium measure of $K$.

The Lelong class $L(X)$ on an $S$-parabolic manifold $X$ with a parabolic potential $\tau$ is defined to be the class of psh functions $u$ on $X$ satisfying the growth condition $u \leq \tau+C$ on $X$ for some constant $C>0$ depending on $u$, see for example [1, 2, 3, 22, 23, 24, 25]. The extremal function and the equilibrium measure for a compact set $K \subset X$ is then defined analogously 
as in the case when $X=\mathbb{C}^{n}$, with $L\left(\mathbb{C}^{n}\right)$ replaced by $L(X)$. In the case of the torus $X=\mathbb{C}^{n} / \mathbb{Z}^{n}$, it is simple to show that $\tau(z):=|\operatorname{Im}(z)|$ is a parabolic potential, so $L\left(\mathbb{C}^{n} / \mathbb{Z}^{n}\right)$ is the class of psh functions $u$ satisfying the growth condition $u(z)-|\operatorname{Im}(z)| \leq O(1)$ as $|\operatorname{Im}(z)| \rightarrow \infty$.

Denote by $\omega$ the Fubini-Study metric on $\mathbb{P}^{n}(\mathbb{C})$. If we imbed $\mathbb{C}^{n}$ into $\mathbb{P}^{n}(\mathbb{C})$ in the canonical way $\left(z_{1}, \ldots, z_{n}\right) \mapsto\left[1: z_{1}: \cdots: z_{n}\right]$ then $\omega$ has a psh potential on $\mathbb{C}^{n}$, namely

$$
\omega=\frac{1}{2} d d^{c} \log \left(1+|z|^{2}\right), \quad z \in \mathbb{C}^{n} .
$$

We define the class $\operatorname{PSH}\left(\mathbb{P}^{n}(\mathbb{C}), \omega\right)$ of $\omega$-psh functions on $\mathbb{P}^{n}(\mathbb{C})$ as the set of all $\varphi \in L^{1}\left(\mathbb{P}^{n}(\mathbb{C})\right)$, which can locally be written as the sum of a smooth function and a psh function and $d d^{c} \varphi+\omega \geq 0$. For a compact set $K \subset \mathbb{P}^{n}(\mathbb{C})$ we define the $\omega$-extremal function for $K$ to be

$$
V_{K, \omega}(z)=\sup \left\{\varphi(z) ; \varphi \in \operatorname{PSH}\left(\mathbb{P}^{n}(\mathbb{C}), \omega\right),\left.\varphi\right|_{K} \leq 0\right\}
$$

and the $\omega$-equilibrium measure for $K$ is $\left(d d^{c} V_{K, \omega}\right)^{n}$. For a detailed survey on $\omega$-psh functions we refer to Guedj and Zeriahi [15]. See also Magnússon [19, 20].

Compact subsets of $\mathbb{R}^{n} \subset \mathbb{C}^{n}$. Although Theorem 1.1 can be applied to calculate the extremal function for a variety of compact subsets of $\mathbb{C}^{n}$ we restrict our attention to compact subsets of $\mathbb{R}^{n}$ simply because authors in the past have shown particular interest in this case.

Lemma 4.1. Let $f=\left(f_{0}, \ldots, f_{n}\right)$ be a holomorphic map on $U \subset \mathbb{C}^{n}$ satisfying $f_{0}+\cdots+f_{n}=1$ and write

$$
K=\left\{z \in U ; f_{j}(z) \in \mathbb{R}_{+} \text {for all } j\right\} .
$$

Then the function

$$
\begin{aligned}
\psi: & =2 \log \left|\left(\sqrt{f_{0}}, \ldots, \sqrt{f_{n}}\right)\right|_{c} \\
& =\log \left(\left|f_{0}\right|+\cdots+\left|f_{n}\right|+\left(\left(\left|f_{0}\right|+\cdots+\left|f_{n}\right|\right)^{2}-1\right)^{1 / 2}\right)
\end{aligned}
$$

equals 0 on $K$ and is maximal on $U \backslash K$. We have

$$
\left.\left(d d^{c} \psi\right)^{n}\right|_{K}= \pm \frac{n ! \Omega_{n}}{\sqrt{f_{0} \cdots f_{n}}} d f_{1} \wedge \cdots \wedge d f_{n}
$$

Proof. It is easy to see that $\psi=0$ on $K$. First we show that $K=$ $f^{-1}\left(\mathbb{C R}_{+}^{n+1}\right)$, where $\mathbb{C R}_{+}^{n+1}=\left\{\alpha a ; \alpha \in \mathbb{C}, a \in[0,+\infty)^{n+1}\right\}$. We clearly 
have $K \subset f^{-1}\left(\mathbb{C R}_{+}^{n+1}\right)$. Conversely, if $z \in f^{-1}\left(\mathbb{C R}_{+}^{n+1}\right)$ there exist positive real numbers $x_{0}, \ldots, x_{n}$ and $\theta \in[0,2 \pi]$ such that

$$
f_{0}(z)=x_{0} e^{i \theta}, f_{1}(z)=x_{1} e^{i \theta}, \ldots, f_{n}(z)=x_{n} e^{i \theta} .
$$

Adding all the equations gives

$$
1=f_{0}(z)+\cdots+f_{n}(z)=e^{i \theta}\left(x_{0}+\cdots+x_{n}\right)
$$

so $e^{i \theta}=1$ which means that $f(z) \in \mathbb{R}_{+}^{n+1}$ so $z \in K$. Now we apply Theorem 1.1 with $\Phi=\left(\sqrt{f_{0}}, \ldots, \sqrt{f_{n}}\right)$. We clearly have $\Phi^{-1}\left(\mathbb{C R}^{n+1}\right)=$ $f^{-1}\left(\mathbb{C R}_{+}^{n+1}\right)=K$ and since $\Phi$ is a holomorphic map in a neighborhood of any point outside the set $S:=\left\{z \in U ; f_{0}(z) \cdots f_{n}(z)=0\right\}$ we see that the function $\psi=\log |\Phi|_{c}^{2}$ is psh and maximal on $U \backslash(K \cup S)$. But since $S$ is pluripolar we can conclude that $\psi$ is psh and maximal on $U \backslash K$. By simply substituting $\Phi_{j}$ with $\sqrt{f_{j}}$ in equation 1.1 from Theorem 1.1 we get the equation

$$
\left.\left(d d^{c} \psi\right)^{n}\right|_{K}= \pm \frac{n ! \Omega_{n}}{\sqrt{f_{0} \cdots f_{n}}}\left(\sum_{j=0}^{n}(-1)^{j+1} f_{j} \Lambda_{f_{j}}\right) .
$$

Using the assumption $f_{0}+\cdots+f_{n}=1$ it is easy to show that

$$
\sum_{j=0}^{n}(-1)^{j+1} f_{j} \Lambda_{f_{j}}=d f_{1} \wedge \cdots \wedge d f_{n}
$$

and equation (4.1) follows.

At this point we should point out the similarities to the Joukovski transformation $J: \mathbb{C} \rightarrow \mathbb{C}$ defined by the formula $J(z)=\frac{1}{2}\left(z+\frac{1}{z}\right)$. It maps $\mathbb{C} \backslash \overline{\mathbb{D}}(0,1)$ bijectively to $\mathbb{C} \backslash[-1,1]$ and its inverse is given with the formula

$$
J^{-1}(z)=z+\left(z^{2}-1\right)^{1 / 2}
$$

where the square root is chosen such that $J^{-1}(x)>1$ for $x>1$. The function $\psi$ from Lemma 4.1 can now be expressed as

$$
\psi(z)=\log J^{-1}\left(\left|f_{0}\right|+\cdots+\left|f_{n}\right|\right) .
$$

We therefore see that our results, along with the extra condition $f_{0}+\cdots+$ $f_{n}=1$ brings us to a familiar territory, as the Joukovski map has been extensively studied for a long time. We include three examples which show the simplicity of this application of Theorem 1.1. Two of them can be found in Klimek [17, Section 5.4.

Example 4.2. (The simplex). It is easy to see that

$$
K:=\left\{z \in \mathbb{C}^{n} ; z_{j} \in \mathbb{R}_{+} \text {, for all } j \text { and } 1-z_{1}-\cdots-z_{n} \in \mathbb{R}_{+}\right\}
$$


is the simplex in $\mathbb{R}^{n} \subset \mathbb{C}^{n}$. By applying Lemma 4.1 we see that the extremal function for $K$ is $V_{K}(z)=\log J^{-1}\left(\left|f_{0}\right|+\cdots+\left|f_{n}\right|\right)$ where

$$
f_{0}(z)=1-z_{1}-\cdots-z_{n}, \quad \text { and } \quad f_{j}(z)=z_{j}, \quad 1 \leq j \leq n .
$$

Using equation (4.1) we see that the equilibrium measure for $K$ is

$$
\left.\left(d d^{c} V_{K}\right)^{n}\right|_{K}=\frac{n ! \Omega_{n}}{\sqrt{x_{1} \cdots x_{n} \cdot\left(1-x_{1}-\cdots-x_{n}\right)}} d x_{1} \wedge \cdots \wedge d x_{n} .
$$

Example 4.3. (The unit ball). Let

$$
K=\left\{x \in \mathbb{R}^{n} ; 1-x_{1}^{2}-\cdots-x_{n}^{2} \geq 0\right\}
$$

be the unit ball in $\mathbb{R}^{n} \subset \mathbb{C}^{n}$. By Lemma 4.1 we see that the extremal function for $K$ is $V_{K}(z)=\log J^{-1}\left(\left|f_{0}\right|+\cdots+\left|f_{n}\right|\right)$ where

$$
f_{0}(z)=1-z_{1}^{2}-\cdots-z_{n}^{2}, \quad \text { and } \quad f_{j}(z)=z_{j}^{2}, \quad 1 \leq j \leq n .
$$

Using equation (4.1) we see that the equilibrium measure for $K$ is

$$
\left.\left(d d^{c} V_{K}\right)^{n}\right|_{K}=\frac{2^{n} n ! \Omega_{n}}{\sqrt{1-x_{1}^{2}-\cdots-x_{n}^{2}}} d x_{1} \wedge \cdots \wedge d x_{n} .
$$

Example 4.4. (The first and third quadrant of a disk). Let

$$
K=\left\{\left(x_{1}, x_{2}\right) \in \mathbb{R}^{2} ; 1-x_{1}^{2}-x_{2}^{2} \geq 0, x_{1} \cdot x_{2} \geq 0\right\}
$$

be the union of the first and third quadrant of the unit disk in $\mathbb{R}^{2} \subset \mathbb{C}^{2}$. It is easy to show that

$$
K=\left\{z \in \mathbb{C}^{2} ; 1-z_{1}^{2}-z_{2}^{2} \in \mathbb{R}_{+}, \quad\left(z_{1}-z_{2}\right)^{2} \in \mathbb{R}_{+}, \quad 2 z_{1} z_{2} \in \mathbb{R}_{+}\right\},
$$

and therefore $V_{K}(z)=\log J^{-1}\left(\left|f_{0}\right|+\left|f_{1}\right|+\left|f_{2}\right|\right)$ where

$$
f_{0}(z)=1-z_{1}^{2}-z_{2}^{2}, \quad f_{1}(z)=\left(z_{1}-z_{2}\right)^{2} \quad \text { and } \quad f_{2}(z)=2 z_{1} z_{2} .
$$

By equation (4.1) we see that

$$
\left.\left(d d^{c} V_{K}\right)^{2}\right|_{K}=\frac{4 \sqrt{2} \pi\left|x_{1}+x_{2}\right|}{\sqrt{x_{1} x_{2}\left(1-x_{1}^{2}-x_{2}^{2}\right)}} d x_{1} \wedge d x_{2} .
$$

Compact subsets of the complex projective space $\mathbb{P}^{n}(\mathbb{C})$. We denote by $\pi: \mathbb{C}^{n+1} \backslash\{0\} \rightarrow \mathbb{P}^{n}(\mathbb{C})$ the usual projection $\pi\left(z_{0}, \ldots, z_{n}\right)=\left[z_{0}: \cdots: z_{n}\right]$. We start with a lemma very similar to Lemma 4.1.

Lemma 4.5. Let $f_{0}, \ldots, f_{n}$ be homogeneous polynomials in $\mathbb{C}^{n+1}$ of degree $2 k$ satisfying the equation $f_{0}+\cdots+f_{n}=\left(z_{0}^{2}+\cdots+z_{n}^{2}\right)^{k}$ and write

$$
K=\pi\left(\left\{z \in \mathbb{C}^{n+1} \backslash\{0\} ;\left(f_{0}(z), \ldots, f_{n}(z)\right) \in \mathbb{R}_{+}^{n+1}\right\}\right) \subset \mathbb{P}^{n}(\mathbb{C}) .
$$

Then the function

$$
\psi:=\log \left|\left(\sqrt{f_{0}}, \ldots, \sqrt{f_{n}}\right)\right|_{c}^{1 / k}-\log \left|\left(z_{0}, \ldots, z_{n}\right)\right|
$$


equals 0 on $K$ and $\left(d d^{c} \psi+\omega\right)^{n}=0$ on $\mathbb{P}^{n}(\mathbb{C}) \backslash K$. We have

$$
\left.\left(d d^{c} \psi+\omega\right)^{n}\right|_{K}=\frac{n ! \Omega_{n}}{2^{n}\left|z_{0}^{2}+\cdots+z_{n}^{2}\right|^{\frac{k(n+1)}{2}} \sqrt{f_{0} \cdots f_{n}}}\left(\sum_{j=0}^{n}(-1)^{j+1} f_{j} \Lambda_{f_{j}}\right) .
$$

Proof. It is simple to show that

$$
\begin{aligned}
\mathbb{C} \cdot\left\{z \in \mathbb{C}^{n+1} ;\right. & \left.\left(f_{0}(z), \ldots, f_{n}(z)\right) \in \mathbb{R}_{+}^{n+1}\right\} \\
& =\left\{z \in \mathbb{C}^{n+1} ;\left(f_{0}(z), \ldots, f_{n}(z)\right) \in \mathbb{C R}_{+}^{n+1}\right\}
\end{aligned}
$$

and therefore

$$
K=\pi\left(\left\{z \in \mathbb{C}^{n+1} \backslash\{0\} ;\left(f_{0}(z), \ldots, f_{n}(z)\right) \in \mathbb{C R}_{+}^{n+1}\right\}\right) .
$$

The rest of the proof is almost identical to the one of Lemma 4.1 .

We consider two examples, the first one is studied in [9].

Example 4.6. (The real projective space). Let

$$
K=\mathbb{P}^{n}(\mathbb{R})=\left\{\left[x_{0}: \cdots: x_{n}\right] ; x_{j} \in \mathbb{R} \text { for all } j\right\} \subset \mathbb{P}^{n}(\mathbb{C})
$$

be the real projective space. It is easy to see that

$$
K=\pi\left(\left\{z \in \mathbb{C}^{n+1} \backslash\{0\} ; z_{j}^{2} \in \mathbb{R}_{+} \text {for all } j\right\}\right)
$$

and therefore

$$
V_{K, \omega}(z)=\log \left|\left(z_{0}, \ldots, z_{n}\right)\right|_{c}-\log \left|\left(z_{0}, \ldots, z_{n}\right)\right|, \quad z=\left[z_{0}: \cdots: z_{n}\right],
$$

is the $\omega$-extremal function for $K$. We clearly have $K \cap \mathbb{C}^{n}=\mathbb{R}^{n}$ and

$$
\left.\left(d d^{c} V_{K, \omega}+\omega\right)^{n}\right|_{\mathbb{R}^{n}}=\frac{n ! \Omega_{n}}{\left(1+x_{1}^{2}+\cdots+x_{n}^{2}\right)^{\frac{(n+1)}{2}}} d x_{1} \wedge \cdots \wedge d x_{n} .
$$

Example 4.7. (The first and third quadrant of the plane). Let

$$
K=\pi\left(\left\{\left(x_{0}, x_{1}, x_{2}\right) \in \mathbb{R}^{3} \backslash\{0\} ; x_{1} \cdot x_{2} \geq 0\right\}\right) \subset \mathbb{P}^{2}(\mathbb{C}) .
$$

When restricted to $\mathbb{C}^{2}$ the set $K$ becomes the union of the first and third quadrant of the plane $\mathbb{R}^{2}$, i.e.

$$
K \cap \mathbb{C}^{2}=\left\{\left(x_{1}, x_{2}\right) \in \mathbb{R}^{2} ; x_{1} \cdot x_{2} \geq 0\right\} .
$$

It is relatively simple to show that $K$ can be represented as

$$
K=\pi\left(\left\{\left(z_{0}, z_{1}, z_{2}\right) \in \mathbb{C}^{3} \backslash\{0\} ; z_{0}^{2} \in \mathbb{R}_{+},\left(z_{1}-z_{2}\right)^{2} \in \mathbb{R}_{+}, 2 z_{1} z_{2} \in \mathbb{R}_{+}\right\}\right)
$$

and therefore the $\omega$-extremal function for $K$ is

$$
V_{K, \omega}(z)=\log \left|\left(z_{0},\left(z_{1}-z_{2}\right), \sqrt{2 z_{1} z_{2}}\right)\right|_{c}-\log \left|\left(z_{0}, z_{1}, z_{2}\right)\right|, \quad z=\left[z_{0}, z_{1}, z_{2}\right] .
$$


We have

$$
\left.\left(d d^{c} V_{K, \omega}+\omega\right)^{2}\right|_{K \cap \mathbb{C}^{2}}=\frac{\sqrt{2} \pi\left(x_{1}+x_{2}\right)}{\left(1+x_{1}^{2}+x_{2}^{2}\right)^{\frac{3}{2}} \sqrt{x_{1} x_{2}}} d x_{1} \wedge d x_{2} .
$$

A compact subset of the complex torus. Finally we consider one compact subset of the complex torus $X=\mathbb{C}^{n} / \mathbb{Z}^{n}$. Define the functions $f_{0}, \ldots, f_{n}$ on $X$ by

$$
f_{0}(z)=\frac{1}{n} \sum_{j=1}^{n} \cos ^{2}\left(\pi z_{j}\right), \quad z=\left(z_{1}, \ldots, z_{n}\right) \in X
$$

and

$$
f_{j}(z)=\frac{1}{n} \sin ^{2}\left(\pi z_{j}\right), \quad j \in\{1, \ldots, n\}, z \in X,
$$

so we clearly have $f_{0}+\cdots+f_{n}=1$ on $X$. Then the set

$$
\begin{aligned}
K & =\left\{z \in X ; f_{j}(z) \in \mathbb{R}_{+} \text {for all } j\right\} \\
& =\left\{z \in X ; y_{j}=0 \text { or } x_{j}=1 / 2 \text { for all } j \text { and } \sum_{j=1}^{n} \cos ^{2}\left(\pi z_{j}\right) \geq 0\right\}
\end{aligned}
$$

is easily seen to be compact. By Lemma 4.1 the function

$$
\psi=\log J^{-1}\left(\left|f_{0}\right|+\cdots+\left|f_{n}\right|\right)
$$

is maximal on $X \backslash K$. For $J \subset\{1, \ldots, n\}$ write

$$
K_{J}=K \cap\left\{z \in X ; y_{j}=0 \text { if } j \in J \text { and } x_{j}=1 / 2 \text { if } j \notin J\right\} .
$$

Then we have $K=\cup_{J} K_{J}$ and notice that $K_{\{1, \ldots, n\}}=\mathbb{R}^{n} / \mathbb{Z}^{n}$ and $K_{\emptyset}=\emptyset$. To simplify notation we only calculate $\left(d d^{c} \psi\right)^{n}$ on $K_{J}$ when $J$ is of the type $J=\{1,2, \ldots, s\}$ for some $1 \leq s \leq n$ since every other $K_{J}$ is practically identical to some $K_{J}$ of this type. Indeed if $J=\{1,2, \ldots, s\}$, then

$$
\left.f_{0}\right|_{K_{J}}=\frac{1}{n}\left(\sum_{j=0}^{s} \cos ^{2}\left(\pi x_{j}\right)-\sum_{j=s+1}^{n} \sinh ^{2}\left(\pi y_{j}\right)\right)
$$

and

$$
\left.f_{j}\right|_{K_{J}}=\frac{1}{n} \sin ^{2}\left(\pi x_{j}\right) \text { if } j \leq s,\left.\quad f_{j}\right|_{K_{J}}=\frac{1}{n} \cosh ^{2}\left(\pi y_{j}\right) \text { if } j>s .
$$

By Lemma 4.1 we have

$$
\begin{gathered}
\left.\left(d d^{c} \psi\right)^{n}\right|_{K_{J}}=\frac{2^{n} n ! \pi^{n} \Omega_{n}}{n^{n / 2} \sqrt{f_{0}}}\left|\cos \left(\pi x_{1}\right) \cdots \cos \left(\pi x_{s}\right) \sinh \left(\pi y_{s+1}\right) \cdots \sinh \left(\pi y_{n}\right)\right| \\
d x_{1} \wedge \cdots \wedge d x_{s} \wedge d y_{s+1} \wedge \cdots \wedge d y_{n} .
\end{gathered}
$$




\section{POLYNOMIAL MAPS}

Let $X$ be an $n$ dimensional complex manifold and $q_{0}, \ldots, q_{n-1}$ be holomorphic functions on $X$. For every $z \in X$ denote by $P_{z}: \mathbb{C} \rightarrow \mathbb{C}$ the polynomial defined by the equation

$$
\begin{aligned}
P_{z}(\zeta) & =\zeta^{n+1}-\zeta^{n}+q_{n-1}(z) \zeta^{n-1}-\cdots+(-1)^{n} q_{1}(z) \zeta+(-1)^{n+1} q_{0}(z) \\
& =\zeta^{n+1}-\zeta^{n}+\sum_{k=0}^{n-1}(-1)^{n+1-k} q_{k}(z) \zeta^{k} .
\end{aligned}
$$

For every $z$ denote by $f_{0}(z), \ldots, f_{n}(z)$ the roots of the polynomial $P_{z}$ and define the set

$$
\begin{aligned}
K: & =\left\{z \in \mathbb{C}^{n} ; \text { all the roots of } P_{z} \text { are positive real numbers }\right\} \\
& =\left\{z \in \mathbb{C}^{n} ; f_{j}(z) \in \mathbb{R}_{+}, \quad 0 \leq j \leq n\right\} .
\end{aligned}
$$

Notice that we have $f_{0}+\cdots+f_{n}=1$ so Lemma 4.1 applies in this situation.

Theorem 5.1. Define $\psi: \mathbb{C}^{n} \rightarrow \mathbb{R}$ by $\psi(z)=\log J^{-1}\left(\left|f_{0}\right|+\cdots+\left|f_{n}\right|\right)$. The function $\psi$ equals 0 on $K$, it is maximal on $\mathbb{C}^{n} \backslash K$ and

$$
\left.\left(d d^{c} \psi\right)^{n}\right|_{K}= \pm \frac{n ! \Omega_{n}}{\sqrt{q_{0}(z) \Delta(z)}} d q_{0} \wedge \cdots \wedge d q_{n-1} .
$$

where $\Delta(z)$ is the discriminant of the polynomial $P_{z}$.

Recall that the discriminant of a polynomial $p$ of degree $n$ with roots $s_{1}, \ldots, s_{n}$ and leading coefficient $a_{n}$ is defined as

$$
\Delta:=a_{n}^{2 n-2} \prod_{j<k}\left(s_{j}-s_{k}\right)^{2}=(-1)^{\frac{n(n-1)}{2}} a_{n}^{2 n-2} \prod_{j \neq k}\left(s_{j}-s_{k}\right) .
$$

The discriminant can also be represented in terms of the derivative of $p$, indeed we have

$$
\prod_{j=1}^{n} p^{\prime}\left(s_{j}\right)=\prod_{j=1}^{n}\left(a_{n} \prod_{\substack{k=1 \\ k \neq j}}^{n}\left(s_{k}-s_{j}\right)\right)=\frac{(-1)^{\frac{n(n-1)}{2}} \Delta}{a_{n}^{n-2}} .
$$

It follows easily from definition of the discriminant that for every monic polynomial $p$ of degree $n$ with discriminant $\Delta$ and roots $s_{1}, \ldots, s_{n}$ we have

$$
\tilde{\Delta}_{j} \cdot\left(p^{\prime}\left(s_{j}\right)\right)^{2}=\Delta, \quad j=1, \ldots, n
$$

where $\tilde{\Delta}_{j}$ is the discriminant of $\frac{p(z)}{z-s_{j}}$. Before we prove Theorem 5.1 we consider a lemma. 
Lemma 5.2. Let $P_{z}, q_{0}, \ldots, q_{n-1}, f_{0}, \ldots, f_{n}$ and $\Delta$ be as in Theorem 5.1. Then we have

$$
d f_{1} \wedge \cdots \wedge d f_{n}= \pm \frac{d q_{0} \wedge \cdots \wedge d q_{n-1}}{\sqrt{\Delta(z)}}
$$

Proof. For every $j=0, \ldots, n$ we have

$$
0=P_{z}\left(f_{j}(z)\right)=f_{j}^{n+1}(z)-f_{j}^{n}(z)+\sum_{k=0}^{n-1}(-1)^{n+1-k} q_{k}(z) f_{j}^{k}(z) .
$$

Differentiating equation (5.5) gives

$$
P_{z}^{\prime}\left(f_{j}(z)\right) d f_{j}=\sum_{k=0}^{n-1}(-1)^{n-k} f_{j}^{k}(z) d q_{k} .
$$

Wedging equation (5.6) over $1 \leq j \leq n$ (notice we do not include $j=0$ in the wedge product) we get

$$
\left(\prod_{j=1}^{n} P_{z}^{\prime}\left(f_{j}(z)\right)\right) d f_{1} \wedge \cdots \wedge d f_{n}=(-1)^{\frac{n(n-1)}{2}} \operatorname{det}(A) d q_{0} \wedge \cdots \wedge d q_{n-1}
$$

where $A$ is the matrix with coefficients $A_{j, k}=f_{j}^{k-1}(z)$. But $A$ is a Vandermonde matrix and therefore we see that $(\operatorname{det}(A))^{2}$ equals the discriminant of the polynomial $\frac{P_{z}(\zeta)}{\zeta-f_{0}(z)}$. Now the result follows by multiplying $P_{z}^{\prime}\left(f_{0}(z)\right)$ on both sides of equation (5.7) and then applying equations (5.2) and (5.3).

Proof of Theorem 5.1. The functions $f_{0}, \ldots, f_{n}$ are holomorphic in a neighborhood of any point $z \in \mathbb{C}^{n}$ such that $\Delta(z) \neq 0$. Therefore, by Lemma 4.1 we see that $\psi$ is maximal on

$$
\mathbb{C}^{n} \backslash(K \cup\{\Delta(z)=0\}) .
$$

It is well known that the discriminant is a holomorphic function so the set $\{\Delta(z)=0\}$ is pluripolar and we conclude that $\psi$ is maximal on $\mathbb{C}^{n} \backslash K$. Equation (5.1) follows from equation (4.1) by applying Lemma 5.2 and noticing that $q_{0}=f_{0} \cdots f_{n}$.

Before we calculate an explicit example we prove the following lemma.

Lemma 5.3. The roots of the polynomial $p(z)=z^{3}-z^{2}+a z-b$ are nonnegative real numbers if and only if $a, b, \Delta$ are non-negative real numbers. 
Proof. Let $s_{0}, s_{1}, s_{2}$ be the roots of $p$. First suppose all the roots are nonnegative real numbers. Then we have

$$
z^{3}-z^{2}+a z-b=\left(z-s_{0}\right)\left(z-s_{1}\right)\left(z-s_{2}\right)
$$

and by comparing coefficients we see that $a, b \geq 0$. Also by definition of the discriminant we have $\Delta \geq 0$. Conversely suppose $a, b, \Delta \geq 0$. Since the discriminant is non-negative all the roots $s_{0}, s_{1}, s_{2}$ are real numbers. We have $p(x)<0$ for all $x<0$ and therefore the roots can't be negative.

Remark: A similar statement for higher order polynomials is not true. As a counter-example we can consider the polynomial

$$
p(z)=z^{4}-z^{3}+\frac{9}{4} z^{2}-z+\frac{5}{4}
$$

which has discriminant $\Delta=\frac{289}{16}$. The polynomial $p$ would satisfy the higher order analogue of Lemma 5.3 but it has 4 complex roots, namely

$$
s_{0}=i, s_{1}=-i, s_{2}=\frac{1}{2}-i, s_{3}=\frac{1}{2}+i \text {. }
$$

Example 5.4. We apply Theorem 5.1 with $X=\mathbb{C}^{2}, q_{0}(z)=z_{2}$ and $q_{1}(z)=$ $z_{1}$ so $P_{z}(\zeta)=\zeta^{3}-\zeta^{2}+z_{1} \zeta-z_{2}$. Then, using the equation for the discriminant for third order polynomials, we have

$$
\Delta(z)=z_{1}^{2}-4 z_{1}^{3}-4 z_{2}-27 z_{2}^{2}+18 z_{1} z_{2} .
$$

Using a program we find that

$$
\begin{aligned}
K & =\left\{\left(z_{1}, z_{2}\right) \in \mathbb{C}^{2} ; \text { all the roots of } P_{z} \text { are real and positive }\right\} \\
& =\left\{\left(x_{1}, x_{2}\right) \in \mathbb{R}_{+}^{2} ; \Delta\left(x_{1}, x_{2}\right) \geq 0\right\}
\end{aligned}
$$

looks like this:

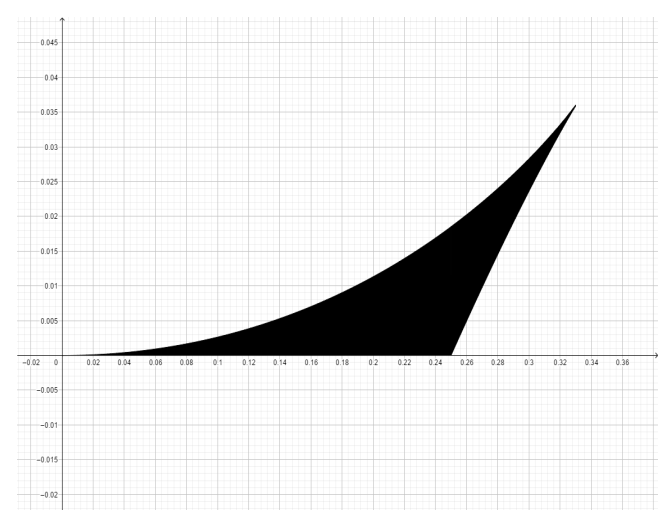


The Monge-Ampere measure of $\psi$ defined as in Theorem 5.1 is

$$
\left.\left(d d^{c} \psi\right)^{2}\right|_{K}=\frac{2 \pi d x_{1} \wedge d x_{2}}{\sqrt{x_{2}\left(x_{1}^{2}-4 x_{1}^{3}-4 x_{2}-27 x_{2}^{2}+18 x_{1} x_{2}\right)}} .
$$

Notice that $\psi$ is not the actual extremal function for $K$ because its growth at infinity differs from the logarithm. This means that we have not calculated the actual equilibrium measure for $K$.

\section{Regularization OF THe Lie NORM}

In this section we study the Levi form of the function $h_{\varepsilon}=\log v_{\varepsilon}$ where $\left(v_{\varepsilon}\right)_{\varepsilon \geq 0}$ is the regularization of $v=|\cdot|_{c}$ given for $\zeta \in \mathbb{C}^{N}$ by

$$
v_{\varepsilon}(\zeta)=\left(|a|^{2}+\varepsilon|b|^{2}\right)^{\frac{1}{2}}+\left(|b|^{2}+\varepsilon|a|^{2}\right)^{\frac{1}{2}}
$$

with the same notation as in the previous sections. We see that $v_{\varepsilon}$ is complex homogeneous of degree $1, v_{\varepsilon}(\zeta) \searrow v(\zeta)=|a|+|b|=|\zeta|_{c}$ as $\varepsilon \searrow 0$. The Levi form of $h_{\varepsilon}$ is quite involved, so in order to simplify our calculations we define the functions $A$ and $B$ on $\mathbb{C}^{N}$ by

$$
A(\zeta)=|a|^{2}=\frac{1}{2}\left(|\zeta|^{2}+|\langle\zeta, \zeta\rangle|\right) \quad \text { and } \quad B(\zeta)=|b|^{2}=\frac{1}{2}\left(|\zeta|^{2}-|\langle\zeta, \zeta\rangle|\right)
$$

and for every $\varepsilon \geq 0, A_{\varepsilon}=A+\varepsilon B$ and $B_{\varepsilon}=B+\varepsilon A$. We observe that

$$
\begin{gathered}
A_{\varepsilon}+B_{\varepsilon}=(1+\varepsilon)(A+B)=(1+\varepsilon)|\zeta|^{2}, \\
A_{\varepsilon}-B_{\varepsilon}=(1-\varepsilon)(A-B)=(1-\varepsilon)|\langle\zeta, \zeta\rangle|, \quad \text { and } \\
4 A_{\varepsilon} B_{\varepsilon}=\left(A_{\varepsilon}+B_{\varepsilon}\right)^{2}-\left(A_{\varepsilon}-B_{\varepsilon}\right)^{2}=(1+\varepsilon)^{2}|\zeta|^{4}-(1-\varepsilon)^{2}|\langle\zeta, \zeta\rangle|^{2} .
\end{gathered}
$$

We define the function $\varphi_{\varepsilon}$ on $\mathbb{C}^{N}$ by

$$
\begin{aligned}
\varphi_{\varepsilon}(\zeta)=2\left(A_{\varepsilon}(\zeta) B_{\varepsilon}(\zeta)\right)^{\frac{1}{2}} & =\left((1+\varepsilon)^{2}|\zeta|^{4}-(1-\varepsilon)^{2}|\langle\zeta, \zeta\rangle|^{2}\right)^{\frac{1}{2}} \\
& =2\left(\left(1+\varepsilon^{2}\right)|a|^{2}|b|^{2}+\varepsilon\left(|a|^{4}+|b|^{4}\right)\right)^{\frac{1}{2}} .
\end{aligned}
$$

The right hand side of 6.2 shows that $\varphi_{\varepsilon}$ is a $C^{\infty}$ function on $\mathbb{C}^{N} \backslash\{0\}$ and the formula for $v_{\varepsilon}$ becomes

$$
v_{\varepsilon}(\zeta)=A_{\varepsilon}^{\frac{1}{2}}+B_{\varepsilon}^{\frac{1}{2}}=\left(A_{\varepsilon}+B_{\varepsilon}+2\left(A_{\varepsilon} B_{\varepsilon}\right)^{\frac{1}{2}}\right)^{\frac{1}{2}}=\left((1+\varepsilon)|\zeta|^{2}+\varphi_{\varepsilon}(\zeta)\right)^{\frac{1}{2}} .
$$

Theorem 6.1. The function $h_{\varepsilon}=\log v_{\varepsilon}$ is psh and maximal on $\mathbb{C}^{N}$. If $\zeta \in \mathbb{C}^{N} \backslash \mathbb{C R}^{N}$ then the Levi-form of $h_{\varepsilon}$ at $\zeta$ is

$$
\mathcal{L}_{h_{\varepsilon}}(\zeta, w)=\frac{(1+\varepsilon)}{2 \varphi_{\varepsilon}(\zeta)}\left(\frac{4 \varepsilon|\zeta|^{4}}{\varphi_{\varepsilon}(\zeta)^{2}}|\langle w, \xi\rangle|^{2}+\left|w^{\prime}\right|^{2}\right)
$$

where $w^{\prime}$ denotes the component of $w$ orthogonal to $\zeta$ and $\bar{\zeta}$, and

$$
\xi=\left(|b| e_{a}-i|a| e_{b}\right) /|\zeta|
$$


is a unit vector in the plane $\operatorname{span}\{\zeta, \bar{\zeta}\}=\operatorname{span}\{a, b\}$ which is perpendicular to $\zeta$, and $e_{a}$ and $e_{b}$ are unit vectors in the direction of $a$ and $b$. In other words, the Levi-matrix of $h_{\varepsilon}$ has three distinct eigenvalues

$$
\lambda_{0}=0, \quad \lambda_{1}=\frac{2 \varepsilon(1+\varepsilon)|\zeta|^{4}}{\varphi_{\varepsilon}(\zeta)^{3}}, \quad \lambda_{2}=\frac{1+\varepsilon}{2 \varphi_{\varepsilon}(\zeta)}
$$

and the corresponding eigenspaces are

$$
V_{0}=\operatorname{span}\{\zeta\}, \quad V_{1}=\operatorname{span}\{\xi\}, \quad V_{2}=\{\zeta, \bar{\zeta}\}^{\perp}=\{a, b\}^{\perp}
$$

Proof. The Levi form of $h_{\varepsilon}$ at $\zeta \in \mathbb{C}^{N} \backslash\{0\}$ is given by

$$
\mathcal{L}_{h_{\varepsilon}}(\zeta ; w)=\frac{1}{v_{\varepsilon}(\zeta)}\left(\mathcal{L}_{v_{\varepsilon}}(\zeta ; w)-\frac{\left|\left\langle\nabla v_{\varepsilon}(\zeta), w\right\rangle\right|^{2}}{v_{\varepsilon}(\zeta)}\right)
$$

where $\nabla v_{\varepsilon}=\left(\partial v_{\varepsilon} / \zeta_{1}, \ldots, \partial v_{\varepsilon} / \zeta_{N}\right)$. Since $v_{\varepsilon}=A_{\varepsilon}^{\frac{1}{2}}+B_{\varepsilon}^{\frac{1}{2}}$, we have

$$
\begin{aligned}
\left\langle\nabla v_{\varepsilon}(\zeta), w\right\rangle & =\frac{1}{2} A_{\varepsilon}^{-\frac{1}{2}}\left\langle\nabla A_{\varepsilon}(\zeta), w\right\rangle+\frac{1}{2} B_{\varepsilon}^{-\frac{1}{2}}\left\langle\nabla B_{\varepsilon}(\zeta), w\right\rangle, \\
\mathcal{L}_{v_{\varepsilon}}(\zeta ; w) & =\frac{1}{2} A_{\varepsilon}^{-\frac{1}{2}} \mathcal{L}_{A_{\varepsilon}}(\zeta ; w)+\frac{1}{2} B_{\varepsilon}^{-\frac{1}{2}} \mathcal{L}_{B_{\varepsilon}}(\zeta ; w) \\
& -\frac{1}{4} A_{\varepsilon}^{-\frac{3}{2}}\left|\left\langle\nabla A_{\varepsilon}(\zeta), w\right\rangle\right|^{2}-\frac{1}{4} B_{\varepsilon}^{-\frac{3}{2}}\left|\left\langle\nabla B_{\varepsilon}(\zeta), w\right\rangle\right|^{2} .
\end{aligned}
$$

Assume now that $\langle\zeta, \zeta\rangle \neq 0$. Then

$$
\frac{\partial|\langle\zeta, \zeta\rangle|}{\partial \zeta_{j}}=\frac{\overline{\langle\zeta, \zeta\rangle}}{|\langle\zeta, \zeta\rangle|} \zeta_{j}=e^{-2 i \theta} \zeta_{j} \quad \text { and } \quad \frac{\partial^{2}|\langle\zeta, \zeta\rangle|}{\partial \zeta_{j} \partial \bar{\zeta}_{k}}=\frac{\zeta_{j} \bar{\zeta}_{k}}{|\langle\zeta, \zeta\rangle|},
$$

so we get

$$
\begin{gathered}
\nabla A(\zeta)=\frac{1}{2}\left(\bar{\zeta}+e^{-2 i \theta} \zeta\right)=e^{-i \theta} a, \quad \nabla B(\zeta)=\frac{1}{2}\left(\bar{\zeta}-e^{-2 i \theta} \zeta\right)=-i e^{-i \theta} b \\
\mathcal{L}_{A}(\zeta ; w)=\frac{1}{2}\left(|w|^{2}+\frac{|\langle\zeta, w\rangle|^{2}}{A-B}\right), \quad \mathcal{L}_{B}(\zeta ; w)=\frac{1}{2}\left(|w|^{2}-\frac{|\langle\zeta, w\rangle|^{2}}{A-B}\right)
\end{gathered}
$$

and consequently

$$
\begin{aligned}
\left\langle\nabla A_{\varepsilon}(\zeta), w\right\rangle & =e^{-i \theta}\langle a-i \varepsilon b, w\rangle, \\
\left\langle\nabla B_{\varepsilon}(\zeta), w\right\rangle & =-i e^{-i \theta}\langle b+i \varepsilon a, w\rangle, \\
\mathcal{L}_{A_{\varepsilon}}(\zeta, w) & =\frac{1}{2}\left((1+\varepsilon)|w|^{2}+(1-\varepsilon) \frac{|\langle\zeta, w\rangle|^{2}}{A-B}\right), \\
\mathcal{L}_{B_{\varepsilon}}(\zeta, w) & =\frac{1}{2}\left((1+\varepsilon)|w|^{2}-(1-\varepsilon) \frac{|\langle\zeta, w\rangle|^{2}}{A-B}\right) .
\end{aligned}
$$

The function $h_{\varepsilon}$ is logarithmically homogeneous, which implies $\mathcal{L}_{h_{\varepsilon}}(\zeta ; \zeta)=0$ and that the Levi matrix of $h_{\varepsilon}$ has 0 eigenvalue with eigenvector $\zeta$.

If we take $w$ orthogonal to both $\zeta$ and $\bar{\zeta}$, i.e., $\langle\zeta, w\rangle=\langle\bar{\zeta}, w\rangle=0$, then $\left\langle\nabla A_{\varepsilon}(\zeta), w\right\rangle=\left\langle\nabla B_{\varepsilon}(\zeta), w\right\rangle=0$, and the formulas 6.6$)-6.12$ give

$$
\mathcal{L}_{h_{\varepsilon}}(\zeta, w)=\frac{(1+\varepsilon)}{4 v_{\varepsilon}(\zeta)}\left(\frac{1}{A_{\varepsilon}(\zeta)^{\frac{1}{2}}}+\frac{1}{B_{\varepsilon}(\zeta)^{\frac{1}{2}}}\right)|w|^{2}=\frac{(1+\varepsilon)}{2 \varphi_{\varepsilon}(\zeta)}|w|^{2} .
$$


From this formula it follows that $\frac{1}{2}(1+\varepsilon) / \varphi_{\varepsilon}(\zeta)$ is an eigenvalue of the Levi matrix and that the eigenspace contains $\{\zeta, \bar{\zeta}\}^{\perp}$, which is of dimension $N-1$ if $\zeta \in \mathbb{C R}^{N}$ and of dimension $N-2$ if $\zeta \in \mathbb{C}^{N} \backslash \mathbb{C R}^{N}$.

Now we assume that $\zeta \in \mathbb{C}^{N} \backslash \mathbb{C R}^{N}$ and let $\xi=\left(|b| e_{a}-i|a| e_{b}\right) /|\zeta|$ be a unit vector in the span of $\zeta$ and $\bar{\zeta}$ orthogonal to $\zeta$. We know that $\xi$ is an eigenvector and the corresponding eigenvalue is $\mathcal{L}_{h_{\varepsilon}}(\zeta, \xi)$. In order to calculate $\mathcal{L}_{h_{\varepsilon}}(\zeta, \xi)$ we first observe that by (6.9)- -6.12$)$ we have

$$
\begin{aligned}
\langle\zeta, \xi\rangle & =\frac{e^{i \theta}}{|\zeta|}\left\langle a+i b,|b| e_{a}-i|a| e_{b}\right\rangle=\frac{2 e^{i \theta}|a||b|}{|\zeta|} \\
\left\langle\nabla A_{\varepsilon}(\zeta), \xi\right\rangle & =\frac{e^{-i \theta}(1-\varepsilon)|a||b|}{|\zeta|}=-\left\langle\nabla B_{\varepsilon}(\zeta), \xi\right\rangle \\
\mathcal{L}_{A_{\varepsilon}}(\zeta, \xi) & =\frac{1}{2}\left(1+\varepsilon+(1-\varepsilon) \frac{4 A B}{A^{2}-B^{2}}\right) \\
\mathcal{L}_{B_{\varepsilon}}(\zeta, \xi) & =\frac{1}{2}\left(1+\varepsilon-(1-\varepsilon) \frac{4 A B}{A^{2}-B^{2}}\right) .
\end{aligned}
$$

By these equations, 6.7), and 6.8) we get

$$
\begin{gathered}
\left|\left\langle\nabla v_{\varepsilon}, \xi\right\rangle\right|^{2}=\frac{A B(1-\varepsilon)^{2}\left(A_{\varepsilon}^{1 / 2}-B_{\varepsilon}^{1 / 2}\right)^{2}}{4(A+B) A_{\varepsilon} B_{\varepsilon}}, \\
\mathcal{L}_{v_{\varepsilon}}(\zeta, \xi)=\frac{(1+\varepsilon) v_{\varepsilon}}{4 A_{\varepsilon}^{1 / 2} B_{\varepsilon}^{1 / 2}}-\frac{(1-\varepsilon) A B\left(A_{\varepsilon}^{1 / 2}-B_{\varepsilon}^{1 / 2}\right)}{\left(A^{2}-B^{2}\right) A_{\varepsilon}^{1 / 2} B_{\varepsilon}^{1 / 2}}-\frac{(1-\varepsilon)^{2} A B\left(A_{\varepsilon}^{3 / 2}+B_{\varepsilon}^{3 / 2}\right)}{4(A+B) A_{\varepsilon}^{3 / 2} B_{\varepsilon}^{3 / 2}} .
\end{gathered}
$$

By equation (6.6) we have

$$
\begin{aligned}
& \mathcal{L}_{h_{\varepsilon}}(\zeta, \xi)=\frac{(1+\varepsilon)}{4 A_{\varepsilon}^{1 / 2} B_{\varepsilon}^{1 / 2}} \\
- & \frac{A B(1-\varepsilon)^{2}}{4(A+B) v_{\varepsilon} A_{\varepsilon}^{1 / 2} B_{\varepsilon}^{1 / 2}}\left(\frac{4\left(A_{\varepsilon}^{1 / 2}-B_{\varepsilon}^{1 / 2}\right)}{A_{\varepsilon}-B_{\varepsilon}}+\frac{A_{\varepsilon}^{3 / 2}+B_{\varepsilon}^{3 / 2}}{A_{\varepsilon} B_{\varepsilon}}+\frac{\left(A_{\varepsilon}^{1 / 2}-B_{\varepsilon}^{1 / 2}\right)^{2}}{A_{\varepsilon}^{1 / 2} B_{\varepsilon}^{1 / 2} v_{\varepsilon}}\right) .
\end{aligned}
$$

The last parenthesis equals $\left(A_{\varepsilon}+B_{\varepsilon}\right)\left(A_{\varepsilon}^{\frac{1}{2}}+B_{\varepsilon}^{\frac{1}{2}}\right) /\left(A_{\varepsilon} B_{\varepsilon}\right)$. Hence

$$
\begin{aligned}
\mathcal{L}_{h_{\varepsilon}}(\zeta, \xi) & =\frac{(1+\varepsilon)}{4 A_{\varepsilon}^{1 / 2} B_{\varepsilon}^{1 / 2}}-\frac{A B(1-\varepsilon)^{2}}{4(A+B) v_{\varepsilon} A_{\varepsilon}^{1 / 2} B_{\varepsilon}^{1 / 2}} \cdot \frac{\left(A_{\varepsilon}+B_{\varepsilon}\right) v_{\varepsilon}}{A_{\varepsilon} B_{\varepsilon}} \\
& =\frac{(1+\varepsilon)}{4 A_{\varepsilon}^{1 / 2} B_{\varepsilon}^{1 / 2}}-\frac{A B(1-\varepsilon)^{2}(1+\varepsilon)}{4 A_{\varepsilon}^{3 / 2} B_{\varepsilon}^{3 / 2}} \\
& =\frac{(1+\varepsilon)}{4\left(A_{\varepsilon} B_{\varepsilon}\right)^{3 / 2}}\left(A_{\varepsilon} B_{\varepsilon}-A B(1-\varepsilon)^{2}\right) \\
& =\frac{\varepsilon(1+\varepsilon)(A+B)^{2}}{4\left(A_{\varepsilon} B_{\varepsilon}\right)^{3 / 2}}=\frac{2 \varepsilon(1+\varepsilon)|\zeta|^{4}}{\varphi_{\varepsilon}(\zeta)^{3}} .
\end{aligned}
$$




\section{PROOF OF THE MAIN RESUlT}

The statement of Theorem 1.1 is local, so without loss of generality we may from now on assume that $X=\mathbb{D}^{n}$, where $\mathbb{D}$ is the unit disc in $\mathbb{C}$. In order to calculate the Monge-Ampère measure of the function $h_{\varepsilon} \circ \Phi$ we need a simple result from linear algebra.

Lemma 7.1. Let $D$ be an $(n+1) \times(n+1)$ Hermitian matrix and $A$ be an $(n+1) \times n$ matrix. Assume that $v$ is a unit eigenvector for $D$ with eigenvalue 0 and denote by $\lambda_{1}, \ldots, \lambda_{n}$ the remaining eigenvalues of $D$. Then

$$
\operatorname{Det}\left(A^{*} D A\right)=\lambda_{1} \cdots \lambda_{n} \cdot|\operatorname{Det}[A \mid v]|^{2}
$$

where $[A \mid v]$ is the $(n+1) \times(n+1)$ matrix obtained by adding $v$ as a columnvector to the right of the matrix $A$.

Proof. By a change of coordinate systems we can assume that $D$ is a diagonal matrix with entries $\lambda_{1}, \ldots, \lambda_{n}$ and 0 , and $v=[0, \ldots, 0,1]^{t}$. Let $\tilde{A}$ be the $n \times n$ matrix obtained by removing the bottom row of $A$ and $\tilde{D}$ be the $n \times n$ diagonal matrix with diagonal $\lambda_{1}, \ldots, \lambda_{n}$. Then $A^{*} D A=\tilde{A}^{*} \tilde{D} \tilde{A}$ and

$$
\operatorname{Det}\left(A^{*} D A\right)=\operatorname{Det}\left(\tilde{A}^{*} \tilde{D} \tilde{A}\right)=\lambda_{1} \cdots \lambda_{n}|\operatorname{Det}(\tilde{A})|^{2}=\lambda_{1} \cdots \lambda_{n}|\operatorname{Det}([A \mid v])|^{2}
$$

by the product formula for determinants.

We have $L_{h_{\varepsilon} \circ \Phi}(z)=J_{\Phi}(z)^{*} L_{h_{\varepsilon}}(\Phi(z)) J_{\Phi}(z)$, where $L_{h_{\varepsilon} \circ \Phi}$ and $L_{h_{\varepsilon}}$ denote the Levi matrices of $h_{\varepsilon} \circ \Phi$ and $h_{\varepsilon}$ respectively. By Theorem 6.1 $L_{h_{\varepsilon}}(\zeta)$ has eigenvalue 0 with eigenvector $\zeta$, and the others are $2 \varepsilon(1+\varepsilon)|\zeta|^{4} / \varphi_{\varepsilon}(\zeta)^{3}$ with multiplicity 1 and $(1+\varepsilon) / 2 \varphi_{\varepsilon}(\zeta)$ with multiplicity $n-1$. Lemma 7.1 gives:

Theorem 7.2. If $\Phi: \mathbb{D}^{n} \rightarrow \mathbb{C}^{n+1} \backslash\{0\}$ is a holomorphic map, then

$$
\left(d d^{c}\left(h_{\varepsilon} \circ \Phi\right)\right)^{n}=\frac{2^{n+2} n !(1+\varepsilon)^{n} \varepsilon|\Phi(z)|^{2}\left|\operatorname{Det}\left[J_{\Phi}(z) \mid \Phi(z)\right]\right|^{2}}{\varphi_{\varepsilon}(\Phi(z))^{n+2}} d V
$$

where $J_{\Phi}$ is the Jacobian of $\Phi$ and $\left[J_{\Phi} \mid \Phi\right]$ is the $(n+1) \times(n+1)$ matrix obtained by adding $\Phi$ as a column vector to the right of $J_{\Phi}$.

It is clear from the definition of $\mathcal{A}_{\Phi}$ in Theorem 1.1 that

$$
\mathcal{A}_{\Phi}=\left\{z \in \mathbb{D}^{n} ; \operatorname{Det}\left[J_{\Phi}(z) \mid \Phi(z)\right]=0\right\} .
$$

The function $z \rightarrow \operatorname{Det}\left[J_{\Phi}(z) \mid \Phi(z)\right]$ is holomorphic so either $\mathcal{A}_{\Phi}$ is pluripolar or it equals $\mathbb{D}^{n}$. If $\mathcal{A}_{\Phi}$ is pluripolar then $\int_{\mathcal{A}_{\Phi}}\left(d d^{c}\left(\log |\Phi|_{c}\right)\right)^{n}=0$ by [17, 
Proposition 4.6.4.]. If $\mathcal{A}_{\Phi}=\mathbb{D}^{n}$ then Theorem 7.2 implies

$$
\left(d d^{c}\left(\log |\Phi|_{c}\right)\right)^{n}=\lim _{\varepsilon \rightarrow 0}\left(d d^{c}\left(h_{\varepsilon} \circ \Phi\right)\right)^{n} \equiv 0 .
$$

From now on we assume that $M=\Phi^{-1}\left(\mathbb{C R}^{n+1}\right) \backslash \mathcal{A}_{\Phi}$ is non-empty. In order to prove that it is a real analytic manifold of dimension $n$ we introduce the auxiliary map $\tilde{\Phi}: \mathbb{D}^{n+1} \rightarrow \mathbb{C}^{n+1}$ defined by the equation

$$
\tilde{\Phi}\left(z, z_{n+1}\right):=\left(1+z_{n+1}\right) \Phi(z), \quad z \in \mathbb{D}^{n}, z_{n+1} \in \mathbb{D} .
$$

Notice that the Jacobians of $\tilde{\Phi}$ and $\Phi$ are related by the equalities

$$
J_{\tilde{\Phi}}=\left[\left(1+z_{n+1}\right) J_{\Phi} \mid \Phi\right], \quad \operatorname{Det} J_{\tilde{\Phi}}=\left(1+z_{n+1}\right)^{n} \operatorname{Det}\left[J_{\Phi} \mid \Phi\right]
$$

and

$$
\tilde{\Phi}^{-1}\left(\mathbb{C} \mathbb{R}^{n+1}\right)=\Phi^{-1}\left(\mathbb{C R}^{n+1}\right) \times \mathbb{D} .
$$

Let $z \in M$ and write $\tilde{z}=(z, 0) \in \mathbb{D}^{n} \times \mathbb{D}$. By equation 7.2 the map $\tilde{\Phi}$ is biholomorphic in a neighborhood of $\tilde{z}$ and the variety $\tilde{\Phi}^{-1}\left(\mathbb{C R}^{n+1}\right)$ has $n+2$ real dimensions in a neighborhood of $\tilde{z}$. By equation 7.3 this means that $M$ has $n$ real dimensions in a neighborhood of $z$.

Denote by

$$
W:=\left\{z \in \mathbb{D}^{n+1} ; \operatorname{Det} J_{\tilde{\Phi}}(z) \neq 0\right\}=\left(\mathbb{D}^{n} \backslash \mathcal{A}_{\Phi}\right) \times \mathbb{D}
$$

the set on which $\tilde{\Phi}$ is locally biholomorphic and thus a submersion. If $z$ is a member of $W$ then the pullback of currents by $\tilde{\Phi}$ at $z$ is well defined and we have the following result.

Theorem 7.3. The $(2 n+2)$-current

$$
\left(d d^{c}(h \circ \Phi)\right)^{n} \wedge \frac{i d z_{n+1} \wedge d \bar{z}_{n+1}}{2\left|1+z_{n+1}\right|^{2}}:=\lim _{\varepsilon \rightarrow 0}\left(d d^{c}\left(h_{\varepsilon} \circ \Phi\right)\right)^{n} \wedge \frac{i d z_{n+1} \wedge d \bar{z}_{n+1}}{2\left|1+z_{n+1}\right|^{2}}
$$

on $W \subset \mathbb{D}^{n+1}$ equals the pullback by $\tilde{\Phi}: \mathbb{D}^{n+1} \rightarrow \mathbb{C}^{n+1}$ of the current of integration along $\mathbb{C R}^{n+1}$ of the $(n+2)$-form $-C_{n}|a|^{-(n+1)} d \theta \wedge d V_{a}$, where $C_{n}=(-1)^{\frac{n(n-1)}{2}} n ! \Omega_{n}$ and $\Omega_{n}$ is the volume of the unit ball in $\mathbb{R}^{n}$.

Proof. To distinguish between them, we now denote by $d V_{n}$ and $d V_{n+1}$ the Euclidean volume forms on $\mathbb{C}^{n}$ and $\mathbb{C}^{n+1}$, respectively. Since the result is local it is sufficient to prove it on $\tilde{\Phi}^{-1}\left(L_{m}\right)$ for some $m \in\{1, \ldots, n+1\}$ where $L_{m}$ is defined in Proposition 3.2. Indeed by Theorem 7.2, homogeneity of 
the function $\varphi_{\varepsilon}$, and by equation 7.2 we have

$$
\begin{aligned}
\left(d d^{c}\left(h_{\varepsilon} \circ \Phi\right)\right)^{n} \wedge \frac{i d z_{n+1} \wedge d \bar{z}_{n+1}}{2\left|1+z_{n+1}\right|^{2}} & =\frac{2^{n+2} n !(1+\varepsilon)^{n} \varepsilon|\Phi|^{2}\left|\operatorname{Det}\left[J_{\Phi} \mid \Phi\right]\right|^{2}}{\left|1+z_{n+1}\right|^{2}\left(\varphi_{\varepsilon} \circ \Phi\right)^{n+2}} d V_{n+1} \\
& =\frac{2^{n+2} n !(1+\varepsilon)^{n} \varepsilon|\tilde{\Phi}|^{2}\left|\operatorname{Det} J_{\tilde{\Phi}}\right|^{2}}{\left(\varphi_{\varepsilon} \circ \tilde{\Phi}\right)^{n+2}} d V_{n+1} \\
& =\tilde{\Phi}^{*}\left(\frac{2^{n+2} n !(1+\varepsilon)^{n} \varepsilon|\zeta|^{2}}{\left(\varphi_{\varepsilon}(\zeta)\right)^{n+2}} d V_{n+1}\right) \\
& =(-1)^{\frac{n(n-1)}{2}} n !(1+\varepsilon)^{n} \tilde{\Phi}^{*}\left(\lambda_{\varepsilon} d \theta \wedge d V_{a} \wedge d V_{\beta}\right),
\end{aligned}
$$

where $\lambda_{\varepsilon}$ is the function defined by the last equality and $\theta, a, \beta$ are variables introduced in Proposition 3.2. Since the pullback is a continuous operation under weak limits it suffices to show that

$$
\lim _{\varepsilon \rightarrow 0}\left(\lambda_{\varepsilon} d \theta \wedge d V_{a} \wedge d V_{\beta}\right)=-\Omega_{n}|a|^{-(n+1)} d \theta \wedge d V_{a} .
$$

By Proposition 3.2 and by equation 6.3 we have

$$
\lambda_{\varepsilon}(\theta, a, \beta)=\frac{-\varepsilon\left(|a|^{2}+|\beta|^{2}\right)\left(|a|-\frac{|\beta|^{2}}{|a|}\right)}{\left(\left(1+\varepsilon^{2}\right)|a|^{2}|\beta|^{2}+\varepsilon\left(|a|^{4}+|\beta|^{4}\right)\right)^{\frac{n+2}{2}}}, \quad(\theta, a, \beta) \in \tilde{L} .
$$

Now let $\chi \in C_{0}^{\infty}\left(\mathbb{C}^{n+1} \backslash\{0\}\right)$ be a test function. We want to show that

$$
\lim _{\varepsilon \rightarrow 0} \int_{|\beta|<|a|} \chi(\theta, a, \beta) \lambda_{\varepsilon}(\theta, a, \beta) d V_{\beta}=-\chi(\theta, a, 0) \Omega_{n}|a|^{-(n+1)} .
$$

Then the result follows by a simple application of Fubini's theorem. We calculate this limit directly by switching into spherical coordinates scaled by a factor of $\varepsilon^{1 / 2}$. We write $r=\varepsilon^{-1 / 2}|\beta|$, we denote by $v$ the unit vector in the direction of $\beta$ and by $d \sigma$ the Euclidean measure on the $(n-1)$ dimensional unit sphere $S^{n-1}$. Hence $\beta=\varepsilon^{1 / 2} r v$ and $d V_{\beta}=\varepsilon^{n / 2} r^{n-1} d r d \sigma$. By the dominated convergence theorem we have

$$
\begin{aligned}
& \lim _{\varepsilon \rightarrow 0} \int_{|\beta|<|a|} \chi(\theta, a, \beta) \lambda_{\varepsilon}(\theta, a, \beta) d V_{\beta} \\
= & \lim _{\varepsilon \rightarrow 0} \int_{\substack{r \in\left(0,|a| \varepsilon^{-1 / 2}\right) \\
v \in S^{n-1}}} \frac{-\varepsilon\left(|a|^{2}+\varepsilon r^{2}\right)\left(|a|-\frac{\varepsilon r^{2}}{|a|}\right) \varepsilon^{\frac{n}{2}} r^{n-1}}{\left(\varepsilon\left(1+\varepsilon^{2}\right)|a|^{2} r^{2}+\varepsilon\left(|a|^{4}+\varepsilon^{2} r^{4}\right)\right)^{\frac{n+2}{2}}} \chi\left(\theta, a, \varepsilon^{1 / 2} r v\right) d r d \sigma \\
= & \int_{\substack{r \in(0, \infty) \\
v \in S^{n-1}}} \frac{-|a|^{3} r^{n-1}}{\left(|a|^{2} r^{2}+|a|^{4}\right)^{1+n / 2}} \chi(\theta, a, 0) d r d \sigma \\
= & -\operatorname{Vol}\left(S^{n-1}\right) \chi(\theta, a, 0)|a|^{-(n-1)} \int_{0}^{\infty} \frac{r^{n-1}}{\left(r^{2}+|a|^{2}\right)^{1+n / 2}} d r \\
= & -\operatorname{Vol}\left(S^{n-1}\right) \chi(\theta, a, 0)|a|^{-(n-1)}\left[\frac{r^{n}}{n|a|^{2}\left(|a|^{2}+r^{2}\right)^{n / 2}}\right]_{0}^{\infty} \\
= & -\Omega_{n} \chi(\theta, a, 0)|a|^{-(n+1)} .
\end{aligned}
$$


We need one more result before we prove Theorem 1.1. To simplify notation we write $\Lambda_{\Phi_{j}}:=d \Phi_{0} \wedge \cdots \wedge \widehat{d \Phi_{j}} \wedge \cdots \wedge d \Phi_{n}$ for $j \in\{0, \ldots, n\}$ and $V:=\tilde{\Phi}^{-1}\left(\mathbb{C R}^{n+1}\right)=\Phi^{-1}\left(\mathbb{C R}^{n+1}\right) \times \mathbb{D}$. Abusing notation a little, for $z=\left(z^{\prime}, z_{n+1}\right) \in \mathbb{D}^{n} \times \mathbb{D}$ we interpret $\Phi(z)$ as $\Phi\left(z^{\prime}\right)$, i.e. we use the same symbol $\Phi$ to denote the trivial extension of $\Phi$ to $\mathbb{D}^{n+1}$.

Proposition 7.4. The restrictions of the $(n+2)$-forms $\tilde{\Phi}^{*}\left(d \theta \wedge d V_{a}\right)$ and

$$
\frac{\left(1+z_{n+1}\right)^{n+1}}{\left|1+z_{n+1}\right|^{2}} e^{-i(n+1) \theta}\left(\sum_{j=0}^{n}(-1)^{j+1} \Phi_{j} \Lambda_{\Phi_{j}}\right) \wedge \frac{i}{2} d z_{n+1} \wedge d \bar{z}_{n+1}
$$

to $V \cap W$ are equal.

Proof. On $V$ the relation between $\Phi_{j}, a_{j}=a_{j} \circ \tilde{\Phi}$ and $\theta=\theta \circ \tilde{\Phi}$ is given by

$$
\left(1+z_{n+1}\right) \Phi_{j}=e^{i \theta} a_{j}, \quad 0 \leq j \leq n,
$$

SO

$$
\Phi_{j} d z_{n+1}+\left.\left(1+z_{n+1}\right) d \Phi_{j}\right|_{V}=i e^{i \theta} a_{j} d \theta+\left.e^{i \theta} d a_{j}\right|_{V} .
$$

The Jacobian of the map $\left.\Phi\right|_{V}: V \rightarrow \mathbb{C R}^{n+1}$ does not have full rank because $\Phi$ is independent of the variable $z_{n+1}$. Therefore $\left.d \Phi_{0} \wedge \cdots \wedge d \Phi_{n}\right|_{V}=0$. Wedging equation (7.4) over all possible $j$ gives

$$
\begin{aligned}
& \left.\left(1+z_{n+1}\right)^{n}\left(\sum_{j=0}^{n}(-1)^{n-j} \Phi_{j} \Lambda_{\Phi_{j}}\right) \wedge d z_{n+1}\right|_{V} \\
& \quad=\left.e^{i(n+1) \theta}\left(d V_{a}+i d \theta \wedge \sum_{j=0}^{n}(-1)^{j} a_{j} \Lambda_{a_{j}}\right)\right|_{V} .
\end{aligned}
$$

Let $0 \leq j_{0} \leq n$ be any fixed number. Similarly as before we have

$$
\bar{\Phi}_{j_{0}} d \bar{z}_{n+1}+\left.\left(1+\bar{z}_{n+1}\right) d \bar{\Phi}_{j_{0}}\right|_{V}=-i e^{-i \theta} a_{j_{0}} d \theta+\left.e^{-i \theta} d a_{j_{0}}\right|_{V} .
$$

Wedging equations 7.6 and (7.5) gives

$$
\begin{aligned}
\bar{\Phi}_{j_{0}}\left(1+z_{n+1}\right)^{n} & \left.\left(\sum_{j=0}^{n}(-1)^{n-j} \Phi_{j} \Lambda_{\Phi_{j}}\right) \wedge d z_{n+1} \wedge d \bar{z}_{n+1}\right|_{V} \\
& =\left.i a_{j_{0}} e^{i n \theta}\left(-d V_{a} \wedge d \theta+(-1)^{j_{0}} d \theta \wedge \Lambda_{a_{j_{0}}} \wedge d a_{j_{0}}\right)\right|_{V} \\
& =\left.2 i(-1)^{n} a_{j_{0}} e^{i n \theta} d \theta \wedge d V_{a}\right|_{V} .
\end{aligned}
$$

After canceling $\left(1+\bar{z}_{n+1}\right) \bar{\Phi}_{j_{0}}=a_{j_{0}} e^{-i \theta}$ the result follows. 
Proof of Theorem 1.1. As we already noted after Theorem 7.2, the MongeAmpère measure has no mass on $\mathcal{A}_{\Phi}$ and $M$ is an $n$ dimensional real analytic manifold, if it is non-empty. The map $\tilde{\Phi}$ is a submersion on $W$. For $\tilde{\Phi} \in$ $\mathbb{C R}^{n+1}$ we have

$$
|a \circ \tilde{\Phi}| e^{i \theta}=|\tilde{\Phi}| e^{i \theta}=\langle\tilde{\Phi}, \tilde{\Phi}\rangle^{\frac{1}{2}}=\left(1+z_{n+1}\right)\langle\Phi, \Phi\rangle^{\frac{1}{2}}
$$

where the complex square root is taken such that $\langle\tilde{\Phi}, \tilde{\Phi}\rangle^{\frac{1}{2}}$ has the same argument $\theta$ as $\tilde{\Phi} \in \mathbb{C}^{n+1}$. By combining Theorem 7.3. Proposition 7.4 and equation 7.8 we see that the $(2 n+2)$-current

$$
\left(d d^{c}(h \circ \Phi)\right)^{n} \wedge \frac{i d z_{n+1} \wedge d \bar{z}_{n+1}}{2\left|1+z_{n+1}\right|^{2}}
$$

on $W$ equals the current of integration along $V$ of the $(n+2)$-form

$$
C_{n}\langle\Phi, \Phi\rangle^{-\frac{n+1}{2}}\left(\sum_{j=0}^{n}(-1)^{j} \Phi_{j} \Lambda_{\Phi_{j}}\right) \wedge \frac{i d z_{n+1} \wedge d \bar{z}_{n+1}}{2\left|1+z_{n+1}\right|^{2}} .
$$

Let $\omega_{1}:=\left(d d^{c}(h \circ \Phi)\right)^{n}$ and define $\omega_{2}$ as the current of integration along $\Phi^{-1}\left(\mathbb{C R}^{n+1}\right)$ of the $n$-form $\sum_{j=0}^{n}(-1)^{j} \Phi_{j} \Lambda_{\Phi_{j}}$. Then

$$
\int_{\mathbb{D}^{n+1}} \chi \omega_{j} \wedge d z_{n+1} \wedge d \bar{z}_{n+1}=\left(\int_{\mathbb{D}^{n}} \chi_{1} \omega_{j}\right)\left(\int_{\mathbb{D}} \chi_{2} d z_{n+1} \wedge d \bar{z}_{n+1}\right),
$$

for $j=1,2$ and $\chi \in C_{0}^{\infty}(W)$ of the form $\chi(z)=\chi_{1}\left(z^{\prime}\right) \chi_{2}\left(z_{n+1}\right)$. This means that we can simply cancel out a factor of $\frac{i}{2} d z_{n+1} \wedge d \bar{z}_{n+1}$ from equations 7.9 and 7.10 when taking the restriction to $W \cap\left\{z_{n+1}=0\right\}=\mathbb{D}^{n} \backslash \mathcal{A}_{\Phi}$.

\section{REFERENCES}

[1] A. Aytuna and A. Sadullaev. $S^{*}$-parabolic manifolds. TWMS J. Pure Appl. Math., $2(1): 6-9,2011$.

[2] A. Aytuna and A. Sadullaev. Parabolic Stein manifolds. Math. Scand., 114(1):86109, 2014.

[3] A. Aytuna and A. Sadullaev. Polynomials on parabolic manifolds. In Topics in several complex variables, volume 662 of Contemp. Math., pages 1-22. Amer. Math. Soc., Providence, RI, 2016.

[4] M. Baran. Plurisubharmonic extremal functions and complex foliations for the complement of convex sets in $\mathbf{R}^{n}$. Michigan Math. J., 39(3):395-404, 1992.

[5] E. Bedford and B. A. Taylor. The Dirichlet problem for a complex Monge-Ampère equation. Invent. Math., 37(1):1-44, 1976.

[6] E. Bedford and B. A. Taylor. A new capacity for plurisubharmonic functions. Acta Math., 149(1-2):1-40, 1982.

[7] E. Bedford and B. A. Taylor. The complex equilibrium measure of a symmetric convex set in $\mathbf{R}^{n}$. Trans. Amer. Math. Soc., 294(2):705-717, 1986.

[8] T. Bloom. Weighted polynomials and weighted pluripotential theory. Trans. Amer. Math. Soc., 361(4):2163-2179, 2009.

[9] L. Bos, N. Levenberg, S. Ma'u, and F. Piazzon. A weighted extremal function and equilibrium measure. Math. Scand., 121(2):243-262, 2017. 
[10] D. Burns, N. Levenberg, and S. Ma'u. Pluripotential theory for convex bodies in $\mathbf{R}^{N}$. Math. Z., 250(1):91-111, 2005.

[11] D. Burns, N. Levenberg, S. Ma'u, and Sz. Révész. Monge-Ampère measures for convex bodies and Bernstein-Markov type inequalities. Trans. Amer. Math. Soc., 362(12):6325-6340, 2010.

[12] D. M. Burns, N. Levenberg, and S. Ma'u. Extremal functions for real convex bodies. Ark. Mat., 53(2):203-236, 2015.

[13] L. M. Drużkowski. Effective formula for the crossnorm in complexified unitary spaces. Zeszyty Nauk. Uniw. Jagiello. Prace Mat., (16):47-53, 1974.

[14] F. Forstnerič. Stein manifolds and holomorphic mappings. Springer, 2nd edition, 2017.

[15] V. Guedj and A. Zeriahi. Degenerate complex Monge-Ampère equations, volume 26 of EMS Tracts in Mathematics. European Mathematical Society (EMS), Zürich, 2017.

[16] L. Hörmander and R. Sigurdsson. Growth properties of plurisubharmonic functions related to Fourier-Laplace transforms. J. Geom. Anal., 8(2):251-311, 1998.

[17] M. Klimek. Pluripotential theory, volume 6 of London Mathematical Society Monographs. New Series. The Clarendon Press, Oxford University Press, New York, 1991. Oxford Science Publications.

[18] M. Lundin. The extremal PSH for the complement of convex, symmetric subsets of $\mathbf{R}^{N}$. Michigan Math. J., 32(2):197-201, 1985.

[19] B.S. Magnússon. Extremal $\omega$-plurisubharmonic functions as envelopes of disc functionals. Ark. Mat., 49(2):383-399, 2011.

[20] B.S. Magnússon. Extremal $\omega$-plurisubharmonic functions as envelopes of disc functionals: generalization and applications to the local theory. Math. Scand., 111(2):296-319, 2012.

[21] G. Munoz, Y. Sarantopoulos, and A. Tonge. Complexifications of real banach spaces, polynomials and multilinear maps. Studia Math., 134:1-33, 1999.

[22] A. S. Snæbjarnarson. Rapid polynomial approximation on Stein manifolds. preprint, arXiv:1612.06173v2, (to appear in Ann. Pol. Math.), 2018.

[23] A. Zeriahi. Fonction de Green pluricomplexe à pôle à l'infini sur un espace de Stein parabolique et applications. Math. Scand., 69(1):89-126, 1991.

[24] A. Zeriahi. Approximation polynomiale et extension holomorphe avec croissance sur une variété algébrique. Ann. Polon. Math., 63(1):35-50, 1996.

[25] A. Zeriahi. A criterion of algebraicity for Lelong classes and analytic sets. Acta Math., 184(1):113-143, 2000.

Department of Mathematics,

School of Engineering and Natural Sciences,

University of Iceland,

IS-107 Reykjavík, ICELAND.

ragnar@hi.is, audunnskuta@hi.is 